\title{
On classical Saito-Kurokawa liftings
}

\author{
By Ralf Schmidt at Norman
}

\begin{abstract}
There exist two different generalizations of the classical Saito-Kurokawa lifting to modular forms with (square-free) level; one lifting produces modular forms with respect to $\Gamma_{0}(m)$, the other one with respect to the paramodular group $\Gamma^{\text {para }}(m)$. We shall give an alternative and unified construction of both liftings using group theoretic methods. The construction shows that a single elliptic modular form may in fact have many SaitoKurokawa liftings. We also obtain precise information about the spin $L$-function of the resulting Siegel modular forms.
\end{abstract}

\section{Introduction}

The classical Saito-Kurokawa lifting was discovered numerically in 1977, and its existence was subsequently proved in a series of papers by Maass, Andrianov and Zagier; see $[\mathrm{Ku}],[\mathrm{Ma}],[\mathrm{An}],[\mathrm{Za}]$. The book $[\mathrm{EZ}]$ gives a coherent treatment. Starting from a modular form $f \in M_{2 k-2}(\mathrm{SL}(2, \mathbb{Z}))$ with even $k$, assumed to be an eigenform for all Hecke operators, a Siegel eigenform $F$ of degree 2 and weight $k$ is constructed such that the (finite parts of the) $L$-functions of $f$ and $F$ are related by the formula

$$
L(s, F)=\zeta(s-k+1) \zeta(s-k+2) L(s, f) .
$$

The Saito-Kurokawa lifting can be constructed as the composition of two linear maps

$$
M_{2 k-2}(\mathrm{SL}(2, \mathbb{Z})) \stackrel{\sim}{\rightarrow} J_{k, 1} \rightarrow M_{k}(\operatorname{Sp}(4, \mathbb{Z})),
$$

where $J_{k, 1}$ is the space of Jacobi forms of weight $k$ and index 1 ; see [EZ]. The first map is obtained via the Shimura isomorphism, and the second map is called the Maaß lifting. In what follows we shall restrict our attention to cusp forms and also to newforms. Given a positive integer $m$, we can generalize the construction (1) as follows:

$$
S_{2 k-2}^{\text {new }-}\left(\Gamma_{0}(m)\right) \stackrel{\sim}{\rightarrow} J_{k, m}^{\text {cusp, new }} \rightarrow S_{k}\left(\Gamma^{\text {para }}(m)\right) .
$$

Here the first isomorphism is the (inverse of the) Skoruppa-Zagier map constructed in [SZ]. The "-" in $S_{2 k-2}^{\text {new- }}\left(\Gamma_{0}(m)\right)$ indicates the subspace of newforms such that the sign in the functional equation of the $L$-function is -1 . The second map in (2) is Gritsenko's "arithmetical lifting", a generalization of the Maaß lifting; see [Gr]. The image is contained in the 
space of cusp forms with respect to the paramodular group $\Gamma^{\text {para }}(m)$ of level $m$. This group is defined as the subgroup of all elements $g \in \operatorname{Sp}(4, \mathbb{Q})$ such that

$$
g \in\left(\begin{array}{cccc}
\mathbb{Z} & m \mathbb{Z} & \mathbb{Z} & \mathbb{Z} \\
\mathbb{Z} & \mathbb{Z} & \mathbb{Z} & m^{-1} \mathbb{Z} \\
\mathbb{Z} & m \mathbb{Z} & \mathbb{Z} & \mathbb{Z} \\
m \mathbb{Z} & m \mathbb{Z} & m \mathbb{Z} & \mathbb{Z}
\end{array}\right) \text { and } \operatorname{det}(g)=1 .
$$

At least when $m$ is square-free (but see the recent work [MR1], [MR2]), there is another generalization of the construction (1) given in the paper [MRV]. Instead of working with Jacobi forms of index $m$ for the full modular group, the authors consider Jacobi forms of index 1 for a congruence subgroup and construct a lifting

$$
S_{2 k-2}^{\text {new }}\left(\Gamma_{0}(m)\right) \stackrel{\sim}{\rightarrow} J_{k, 1}^{\text {cusp, new }}\left(\Gamma_{0}^{J}(m)\right) \rightarrow S_{k}\left(\Gamma_{0}^{(2)}(m)\right) .
$$

For this construction to work $k$ must assumed to be even. Note that both constructions (2) and (4) generalize the original lifting (1). The conditions " $k$ even" and "the sign in the functional equation is -1 " coincide for $m=1$.

Hence, starting with a newform $f \in S_{2 k-2}^{\text {new- }}\left(\Gamma_{0}(m)\right)$, where $m$ is square-free and $k$ is even, we can construct two Siegel cusp forms $F_{1} \in S_{k}\left(\Gamma^{\text {para }}(m)\right)$ and $F_{2} \in S_{k}\left(\Gamma_{0}^{(2)}(m)\right)$. The purpose of this paper is to give an alternative construction and to "explain" the existence of two different Saito-Kurokawa liftings. It turns out that in some cases $F_{1}$ and $F_{2}$ correspond to two different vectors in the same automorphic representation of $\operatorname{PGSp}(4, \mathbb{A})$, while in other cases $F_{1}$ and $F_{2}$ are vectors in different automorphic representations.

Actually, depending on the number of primes dividing the square-free integer $m$, a modular form $f$ can have many different Saito-Kurokawa liftings. Most of these liftings will be with respect to "mixed" congruence subgroups, where we impose a $\Gamma_{0}$-condition at some places $p \mid m$ and a paramodular condition at the other places. The automorphic representations containing these modular forms are all nearly equivalent (meaning the local components are equivalent at almost every place).

Our approach is based on the main result of [Sch2], which asserts the existence of certain functorial liftings

$$
\text { from } \operatorname{PGL}(2) \times \operatorname{PGL}(2) \text { to } \operatorname{PGSp}(4) \text {. }
$$

Such a lifting is predicted by Langlands functoriality. Let $\pi$ be a cusp form on $\operatorname{PGL}\left(2, \mathbb{A}_{F}\right)$, where $F$ is an arbitrary number field. Let $\Sigma$ be the set of places of $F$ such that the local component $\pi_{v}$ is square-integrable (equivalently, not a principal series representation). For $S \subset \Sigma$ let $\pi_{S}$ be the non-cuspidal automorphic representation of $\operatorname{PGL}\left(2, \mathbb{A}_{F}\right)$ such that the $\pi_{S, v}$ is the trivial representation for $v \notin S$, and the Steinberg representation for $v \in S$. It was proved in [Sch2] that the lifting of $\pi \otimes \pi_{S}$ exists as a discrete series representation on $\operatorname{PGSp}(4, \mathbb{A})$, provided the sign condition $(-1)^{\# S}=\varepsilon(1 / 2, \pi)$ is fulfilled. The lifting is even cuspidal if $S$ is non-empty.

Now let the number field be $\mathbb{Q}$ and let $\pi$ be the cuspidal automorphic representation of $\operatorname{PGL}(2, \mathbb{A})$ corresponding to the classical newform $f \in S_{2 k-2}^{\text {new }}\left(\Gamma_{0}(m)\right)$. Assuming that $m$ 
is square-free, the local components $\pi_{p}$ will be square-integrable precisely if $p \mid m$ or $p=\infty$. We will see that the two liftings (2) and (4) come from two different (non-empty) choices of the set of places $S$. The sign condition $(-1)^{\# S}=\varepsilon(1 / 2, \pi)$ comes down to the "_-" condition in case of the lifting (2), and to the condition that $k$ be even in case of the lifting (4).

The local components of the lifting of $\pi \otimes \pi_{S}$ to $\operatorname{PGSp}(4, \mathbb{A})$ have already been identified as representations in [Sch2]. The bulk of the work of the present paper consists in analyzing these local $p$-adic representations at the primes $p$ dividing $m$ with respect to their paramodular and $\Gamma_{0}$-fixvectors. This will allow us to extract from the automorphic representations we constructed the Siegel modular forms we are interested in. To analyze the local representations, we realize them as subrepresentations of certain induced representations of length 2. The spaces of Iwahori fixed vectors on the full induced representations are easily determined. To find the intersections with the subrepresentations of interest, we shall explicitly compute intertwining operators on the spaces of Iwahori fixed vectors.

Two remarks are in order, the first one concerning certain assumptions that had been made in [Sch2]. The construction in [Sch2] was carried out using theta liftings. To prove functoriality, certain plausible assumptions on the still conjectural local Langlands correspondence for GSp(4) had to be made. However, in the present paper we are not concerned with functoriality questions, so the results are independent of any conjectures.

The second remark concerns the paper [Sch3] and its relationship with the present work. While the paper at hand deals with a small class of modular forms of square-free level - the Saito-Kurokawa liftings - the work [Sch3] considers general modular forms of square-free level (with respect to various congruence subgroups). Accordingly, only a handful of Iwahori-spherical representations of $\mathrm{GSp}(4)$ play a role in the current paper, while all such representations had to be considered in [Sch3]. For example, [Sch3], Table 3 gives the dimensions of all spaces of fixed vectors of all Iwahori-spherical representations under all parahoric subgroups. However, to obtain this table, certain special cases had to be treated first, and these are the cases treated in the present work. In this sense [Sch3] depends on the calculations in the present paper.

In section 1 we shall review the main lifting theorem of [Sch2], which lies at the heart of our construction. In sections 2 and 3 we shall analyze the relevant local representations by explicitly computing intertwining operators on certain spaces of induced representations. Actually we shall determine the dimensions of spaces of fixed vectors for each parahoric subgroup of $\operatorname{GSp}(4, F)$. In section 4 we compute some Atkin-Lehner eigenvalues on the "local newforms" we found and show that these eigenvalues coincide with the signs defined by the $\varepsilon$-factor, as in the GL(2) theory. Finally, in section 5 , we combine the global lifting theorem with the local results to reprove the existence of the two different Saito-Kurokawa liftings mentioned above - and others. We also obtain precise information on the spin $L$-function of these liftings.

\section{Notations}

Throughout the paper we let

$$
G=\mathrm{GSp}(4)=\left\{g \in \mathrm{GL}(4):{ }^{t} g J g=\lambda(g) J \text { for some } \lambda(g) \in \mathrm{GL}(1)\right\}
$$


where

$$
J=\left(\begin{array}{llll} 
& & & 1 \\
& & 1 & \\
-1 & & &
\end{array}\right)
$$

As a Borel subgroup $B$ of $G$ we choose upper triangular matrices. The two conjugacy classes of proper maximal parabolic subgroups are represented by the Siegel parabolic subgroup $P$, whose Levi factor is

$$
M_{P}=\left\{\left(\begin{array}{cc}
A & \\
& u A^{\prime}
\end{array}\right): u \in \mathrm{GL}(1), A \in \mathrm{GL}(2)\right\} \simeq \mathrm{GL}(1) \times \mathrm{GL}(2),
$$

where $A^{\prime}:=\left(\begin{array}{ll} & 1 \\ 1 & \end{array}\right){ }^{t} A^{-1}\left(\begin{array}{ll} & 1 \\ 1 & \end{array}\right)$, and the Klingen parabolic subgroup $Q$, whose Levi fac-
tor is

$$
M_{Q}=\left\{\left(\begin{array}{lll}
u & & \\
& A & \\
& & u^{-1} \operatorname{det}(A)
\end{array}\right): u \in \mathrm{GL}(1), A \in \mathrm{GL}(2)\right\} \simeq \mathrm{GL}(1) \times \mathrm{GL}(2) .
$$

If $F$ is a local field, we shall employ the notations of [ST] for induced representations of the group $\operatorname{GSp}(4, F)$. We shall write $v(x)=|x|$ for the normalized absolute value on the local field $F$.

Parahoric subgroups. Let $F$ be a $p$-adic field, $\mathfrak{o}$ its ring of integers, and $\varpi$ a generator of the maximal ideal $\mathfrak{p}$ of $\mathfrak{v}$. The two matrices

$$
s_{1}=\left(\begin{array}{cccc} 
& 1 & & \\
1 & & & \\
& & & 1 \\
& & 1 &
\end{array}\right), \quad s_{2}=\left(\begin{array}{cccc}
1 & & & \\
& & 1 & \\
& -1 & & \\
& & & 1
\end{array}\right)
$$

are representatives for the two simple reflections generating the 8-element Weyl group $W$ of $G=\mathrm{GSp}(4, F)$. The Atkin-Lehner element is the matrix

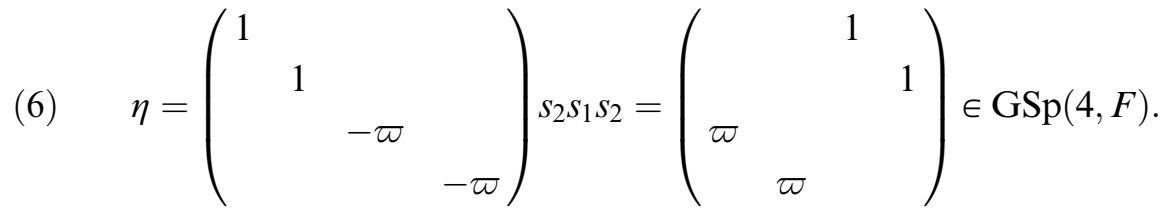

While $\eta$ commutes with $s_{1}$, we let $s_{0}=\eta s_{2} \eta^{-1}$. Consider the Dynkin diagram of the affine Weyl group $C_{2}$ :

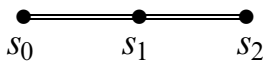


Let $I$ be the Iwahori subgroup, the inverse image of the minimal parabolic subgroup under the projection map $G(\mathfrak{o}) \rightarrow G(\mathfrak{v} / \mathfrak{p})$. The parahoric subgroups $P_{A}$ of $\operatorname{GSp}(4, F)$ correspond bijectively to subsets $A$ of $\{0,1,2\}$, the correspondence being that the parahoric subgroup $P_{A}$ is generated by $I$ and $\left\{s_{i}: i \in A\right\}$. Hence $P_{1}$ is the Siegel congruence subgroup, the inverse image of the Siegel parabolic subgroup under the projection map $G(\mathfrak{v}) \rightarrow G(\mathfrak{v} / \mathfrak{p})$, and $P_{2}$ is the Klingen congruence subgroup, the inverse image of the Klingen parabolic subgroup under the same map. The group $P_{02}$ is generated by $P_{2}$ and $\eta P_{2} \eta^{-1}$, and is called the paramodular group; it is explicitly given as the set of all $g \in \operatorname{GSp}(4, F)$ such that

$$
g \in\left(\begin{array}{cccc}
\mathfrak{D} & \mathfrak{D} & \mathfrak{D} & \mathfrak{p}^{-1} \\
\mathfrak{p} & \mathfrak{D} & \mathfrak{D} & \mathfrak{D} \\
\mathfrak{p} & \mathfrak{D} & \mathfrak{D} & \mathfrak{D} \\
\mathfrak{p} & \mathfrak{p} & \mathfrak{p} & \mathfrak{D}
\end{array}\right) \quad \text { and } \operatorname{det}(g) \in \mathfrak{o}^{*}
$$

This is the local analogue of the global group (3). The paramodular group represents one of the two conjugacy classes of maximal compact subgroups of $\operatorname{GSp}(4, F)$. The other conjugacy class is represented by the standard maximal compact subgroup $P_{12}=G(\mathfrak{v})$.

\section{The main lifting result}

In this section we recall the main result of [Sch2]; we refer to that paper for more details. Let $F$ be a number field and $\mathbb{A}$ its ring of adeles. For each finite set $S$ of places of $F$ we have an automorphic representation $\pi_{S}=\bigotimes \pi_{S, v}$ of $\operatorname{PGL}(2, \mathbb{A})$ defined by

$$
\pi_{S, v}= \begin{cases}\mathbf{1}_{\mathrm{GL}(2)} & \text { if } v \notin S \\ \mathrm{St}_{\mathrm{GL}(2)} & \text { if } v \in S\end{cases}
$$

Here $\mathbf{1}_{\mathrm{GL}(2)}$ is the trivial representation and $\mathrm{St}_{\mathrm{GL}(2)}$ is the Steinberg representation of $\operatorname{PGL}(2)$. The global representation $\pi_{S}$ thus defined is automorphic since it is an irreducible constituent of a globally induced representation.

Let $\pi_{v}$ be an irreducible, admissible, infinite-dimensional representation of the local group $\operatorname{PGL}\left(2, F_{v}\right)$, where $v$ is any place of $F$. We have defined in [Sch2] an irreducible, admissible representation $\Pi\left(\pi_{v} \otimes \mathbf{1}_{v}\right)$ of $\operatorname{PGSp}\left(4, F_{v}\right)$ as the unique irreducible quotient of the induced representation $v^{1 / 2} \pi_{v} \rtimes v^{-1 / 2}$. Assuming that $\pi_{v}$ is square-integrable, we have moreover defined a (tempered) representation $\Pi\left(\pi_{v} \otimes \mathrm{St}_{v}\right)$ of $\operatorname{PGSp}\left(4, F_{v}\right)$ be means of certain theta liftings. If $\pi=\bigotimes \pi_{v}$ is a cuspidal automorphic representation of $\operatorname{PGL}(2, \mathbb{A})$, and $S$ is a set of places such that $\pi_{v}$ is square integrable for each $v \in S$, define a global representation $\Pi\left(\pi \otimes \pi_{S}\right)$ of $\operatorname{PGSp}(4, \mathbb{A})$ as the tensor product $\otimes \Pi\left(\pi_{v} \otimes \pi_{S, v}\right)$.

1.1. Theorem. Let $\pi=\bigotimes \pi_{v}$ be a cusp form on $\operatorname{PGL}(2, \mathbb{A})$. Let $S$ be a set of places of $F$ such that $\pi_{v}$ is a discrete series representation for each place $v \in S$, and let $\pi_{S}$ be the corresponding (non-cuspidal) automorphic representation defined above. Assume that the sign condition

$$
(-1)^{\# S}=\varepsilon(1 / 2, \pi)
$$

is fulfilled. Then: 
(a) The global lifting $\Pi\left(\pi \otimes \pi_{S}\right)$ is an automorphic representation of $\operatorname{PGSp}(4, \mathbb{A})$ which appears discretely in the space of automorphic forms.

(b) If $L(1 / 2, \pi)=0$ or if $S \neq \emptyset$, then $\Pi\left(\pi \otimes \pi_{S}\right)$ is a cuspidal automorphic representation.

Given the results of Waldspurger [Wa1], [Wa2] and Piatetski-Shapiro [PS], this theorem is not too hard to prove. The main point of [Sch2] was to show that if one believes in what is currently conjectured about the local Langlands correspondence for $\operatorname{GSp}(4)$, the representation $\Pi\left(\pi \otimes \pi_{S}\right)$ is a functorial lifting of the representation $\pi \otimes \pi_{S}$ on $\operatorname{PGL}(2, \mathbb{A}) \times \operatorname{PGL}(2, \mathbb{A})$, as the notation suggests.

We now describe the local representations $\Pi\left(\pi_{v} \otimes \pi_{S, v}\right)$ in more detail, starting with the archimedean case. Since we are dealing with classical holomorphic modular forms, let us assume that $F=\mathbb{Q}$, that $v$ is the real place and that $\pi_{v}=\mathscr{D}(2 k-3)$, the holomorphic discrete series representation of $\operatorname{PGL}(2, \mathbb{R})$ with a lowest weight vector of weight $2 k-2$. In our situation we shall always choose $S$ to contain the archimedean place, hence we need only describe $\Pi\left(\pi_{v} \otimes \mathrm{St}_{v}\right)$. Here $\mathrm{St}_{v}$ is the lowest discrete series representation $\mathscr{D}(1)$, and it was determined in [Sch2] (see also [La]) that $\Pi\left(\pi_{v} \otimes \mathrm{St}_{v}\right)$ is the holomorphic discrete series representation of $\operatorname{PGSp}(4, \mathbb{R})$ with a scalar minimal $K$-type of weight $(k, k)$. It is these representations which appear as archimedean components of automorphic representations corresponding to holomorphic Siegel modular forms of weight $k$, see $[\mathrm{AS}]$.

Considering a finite place $p \mid m$, the local component $\pi_{p}$ (of the automorphic representation $\pi$ corresponding to the modular form $f \in S_{2 k-2}^{\text {new }}\left(\Gamma_{0}(m)\right)$, where $m$ is square-free), can only be the Steinberg representation St or its non-trivial unramified twist $\xi \mathrm{St}$; here $\xi$ is the unique non-trivial unramified quadratic character of $\mathbb{Q}_{p}^{*}$. Consequently there are four possible lifts, and these have been identified in [Sch2] as follows.

\begin{tabular}{|l|l|l|}
\hline representation & name & description \\
\hline \hline$\Pi(\mathrm{St} \otimes \mathbf{1})$ & $L\left(\left(v^{1 / 2} \mathrm{St}, v^{-1 / 2}\right)\right)$ & subrepresentation of $\mathbf{1}_{F^{*}} \rtimes \mathbf{1}_{\mathrm{GL}(2)}$ \\
\hline$\Pi(\mathrm{St} \otimes \mathrm{St})$ & $\tau\left(T, v^{-1 / 2}\right)$ & $\begin{array}{c}\text { subrepresentation of } \\
v^{1 / 2} \mathbf{1}_{\mathrm{GL}(2)} \rtimes v^{-1 / 2}\end{array}$ \\
\hline$\Pi(\xi \mathrm{St} \otimes \mathbf{1})$ & $L\left(\left(\xi v^{1 / 2} \mathrm{St}, v^{-1 / 2}\right)\right)$ & $\begin{array}{c}\text { subrepresentation of } \\
\xi v^{1 / 2} \mathbf{1}_{\mathrm{GL}(2)} \rtimes \xi v^{-1 / 2}\end{array}$ \\
\hline$\Pi(\xi \mathrm{St} \otimes \mathrm{St})$ & $\theta_{10}$ & supercuspidal \\
\hline
\end{tabular}

The first three representations in this list are named as in [ST]. In the following sections we shall determine the dimensions of their spaces of fixed vectors under each parahoric subgroup. The fourth representation was investigated in [KPS]. Being supercuspidal it has no Iwahori invariant vectors. In our choices of the set of places $S$ we shall avoid the constellation $\xi \mathrm{St} \otimes$ St. Hence $\theta_{10}$ plays little role in our investigations. 


\section{Invariant vectors in $\Pi(\mathbf{S t} \otimes \mathrm{St})$ and $\Pi(\xi \mathrm{St} \otimes 1)$}

In this section we shall work over a $p$-adic field $F$, with the symbols $\mathfrak{o}, \mathfrak{p}, q$ having the usual meaning. We shall fix a generator $\varpi$ of $\mathfrak{p}$. Consider the degenerate principal series representation

$$
\xi v^{s} \mathbf{1}_{\mathrm{GL}(2)} \rtimes \xi^{-1} v^{-s}, \quad \xi \text { an unramified character of } F^{*}, s \in \mathbb{C} .
$$

Let $V_{\xi, s}$ be the standard model for this induced representation, consisting of smooth functions $f: G(F) \rightarrow \mathbb{C}$ with the transformation property

$$
f\left(\left(\begin{array}{cc}
A & * \\
& u A^{\prime}
\end{array}\right) g\right)=\xi\left(u^{-1} \operatorname{det}(A)\right)\left|u^{-1} \operatorname{det}(A)\right|^{s+3 / 2} f(g) .
$$

According to the table (9), two of our local Saito-Kurokawa lifts appear as constituents of some $V_{\xi, s}$, namely $\Pi(\mathrm{St} \otimes \mathrm{St})$ as a constituent of $V_{1,1 / 2}$, and $\Pi(\xi \mathrm{St} \otimes \mathbf{1})$ as a constituent of $V_{\xi, 1 / 2}$, where $\xi$ is the non-trivial unramified quadratic character of $F^{*}$. To characterize subrepresentations of $V_{\xi, s}$, we shall study an intertwining operator $A(s): V_{\xi, s} \rightarrow V_{\xi^{-1},-s}$, given by

$$
(A(s) f)(g)=\int_{N} f\left(s_{2} s_{1} s_{2} n g\right) d n
$$

where $N=\left(\begin{array}{ll}1 & * \\ & 1\end{array}\right)$ is the unipotent radical of the Siegel parabolic $P$, and where $s_{1}, s_{2}$ are the Weyl group elements defined in (5).

2.1. Proposition. Assume $\xi$ is an unramified character of $F^{*}$. The intertwining operator $A(s)$ defined by (11) is convergent for $\operatorname{Re}(s)$ large enough. It has meromorphic continuation to all of $\mathbb{C}$ with possible poles at those values $s \in \mathbb{C}$ where $q^{-s \pm 1 / 2}=\xi(\varpi)^{-1}$ or where $q^{-2 s}=\xi(\varpi)^{-2}$. At all other values of $s$ it defines a non-zero intertwining map $\xi v^{s} \mathbf{1}_{\mathrm{GL}(2)} \rtimes \xi^{-1} v^{-s} \rightarrow \xi^{-1} v^{-s} \mathbf{1}_{\mathrm{GL}(2)} \rtimes \xi v^{s}$.

Proof. Let $f$ be a function in $V_{\xi, s}$. Let $C_{i} \subset F$ be compact sets containing 0 . One can proof in a standard manner that for large enough $C_{i}$ the integral

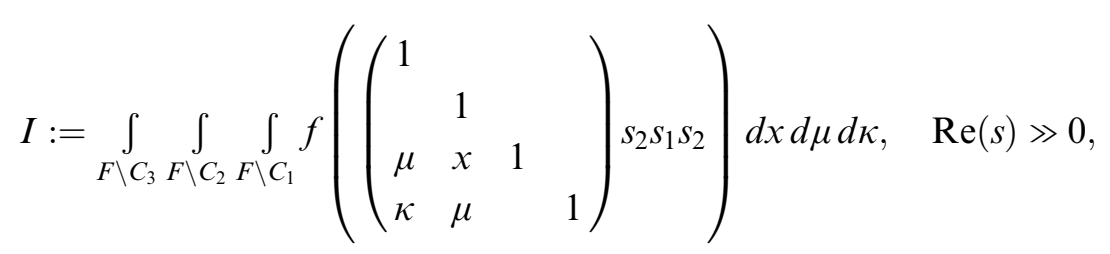

is given by

$$
I=\frac{\text { polynomial function in } q^{-s}}{\left(1-\xi(\varpi) q^{-s+1 / 2}\right)\left(1-\xi(\varpi)^{2} q^{-2 s}\right)\left(1-\xi(\varpi) q^{-s-1 / 2}\right)} f(\mathbf{1}) .
$$

Other parts of the integral, such as $\iiint_{C_{3} \times\left(F \backslash C_{2}\right) \times\left(F \backslash C_{1}\right)} \ldots d x d \mu d \kappa$, lead to similar expressions. This settles the convergence question. Replacing $f$ by a flat section $f_{s}$, it also proves the assertion about analytic continuation. In the region of convergence it is easily seen that $A(s) f_{s}$ has the required transformation property, and that $A(s)$ is an intertwining operator. 
The same is then true for all $s$ by analytic continuation. It will become apparent by the explicit computations further below that $A(s)$ is a non-zero map.

Let $I \subset G(\mathfrak{p})$ be the Iwahori subgroup, i.e., the inverse image of $B(\mathfrak{o} / \mathfrak{p})$ under the projection $G(\mathfrak{v}) \rightarrow G(\mathfrak{v} / \mathfrak{p})$ (in our realization of $G(\mathfrak{v})$, these are all matrices that become upper triangular $\bmod \mathfrak{p})$. Our goal is to determine the space of $I$-invariant vectors in $\Pi(\mathrm{St} \otimes \mathrm{St})$ and $\Pi(\xi \mathrm{St} \otimes \mathbf{1})$, where $\xi$ denotes the unique nontrivial unramified quadratic character of $F^{*}$, characterized by $\xi(\varpi)=-1$. The desired information will be obtained by considering the intertwining operator (11) on the space of $I$-invariant vectors of the degenerate principal series representations $(10)$.

For the moment let $\xi$ be any unramified character of $F^{*}$. As in the previous section, let $V_{\xi, s}$ be the standard space of the induced representation $\xi v^{s} \mathbf{1}_{\mathrm{GL}(2)} \rtimes \xi^{-1} v^{-s}$. Let $V_{\xi, s}^{I}$ denote the subspace of $I$-invariant vectors. Via restriction to $G(\mathfrak{o})$ the functions in $V_{\xi, s}^{I}$ are in bijection with the left $P(\mathfrak{v})$-invariant and right $I$-invariant functions on $G(\mathfrak{v})$. Since

$$
P(\mathfrak{b}) \backslash G(\mathfrak{p}) / I \simeq P(\mathfrak{o} / \mathfrak{p}) \backslash G(\mathfrak{o} / \mathfrak{p}) / B(\mathfrak{o} / \mathfrak{p})
$$

is represented by the four Weyl group elements $\mathbf{1}, s_{2}, s_{2} s_{1}$ and $s_{2} s_{1} s_{2}$, any $f \in V_{\xi, s}^{I}$ is determined by the four numbers

$$
\alpha:=f(\mathbf{1}), \quad \beta:=f\left(s_{2}\right), \quad \gamma:=f\left(s_{2} s_{1}\right), \quad \delta:=f\left(s_{2} s_{1} s_{2}\right) .
$$

In particular, $\operatorname{dim}_{\mathbb{C}}\left(V_{\xi, s}^{I}\right)=4$. We are going to compute $A(s) f$ for such a function $f$. Since $A(s) f$ is again $I$-invariant, we only have to compute $(A(s) f)(w)$ for $w \in\left\{\mathbf{1}, s_{2}, s_{2} s_{1}, s_{2} s_{1} s_{2}\right\}$. This is achieved by the following three lemmas. acter $\xi$,

2.2. Lemma. Let $f \in V_{\xi, s}^{I}$ and $\alpha, \beta, \gamma, \delta$ as in (12). Then, for any unramified char-

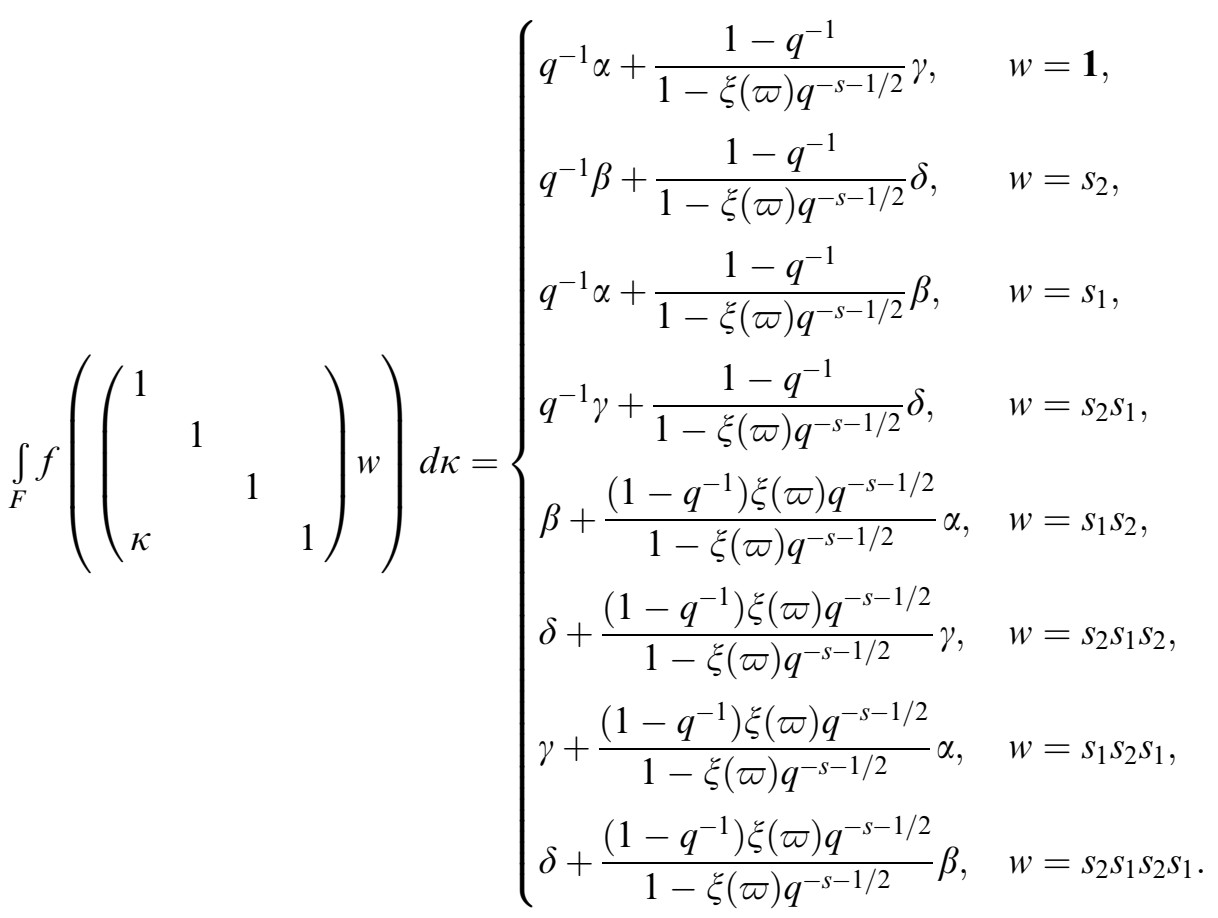


Proof. If the conjugation $g \mapsto w^{-1} g w$ moves the $\kappa$-variable to a positive root, we split the integral as $\int_{\mathfrak{v}}+\int_{F \backslash \mathfrak{D}}$, otherwise as $\int_{\mathfrak{p}}+\int_{F \backslash \mathfrak{p}}$. The first integral can then trivially be computed, while for the second one one uses

$$
\left(\begin{array}{ll}
1 & \\
\kappa & 1
\end{array}\right)=\left(\begin{array}{cc}
-\kappa^{-1} & \\
& -\kappa
\end{array}\right)\left(\begin{array}{ll}
1 & \kappa \\
& 1
\end{array}\right)\left(\begin{array}{cc} 
& 1 \\
-1 &
\end{array}\right)\left(\begin{array}{cc}
1 & \kappa^{-1} \\
& 1
\end{array}\right)
$$

and proceeds in a standard manner.

2.3. Lemma. With $f$ as in Lemma 2.2 define

$$
B(w):=\int_{F^{2}} f\left(\left(\begin{array}{llll}
1 & & & \\
& 1 & & \\
\mu & & 1 & \\
\kappa & \mu & & 1
\end{array}\right) w\right) d \mu d \kappa
$$

Then

$B(w)$

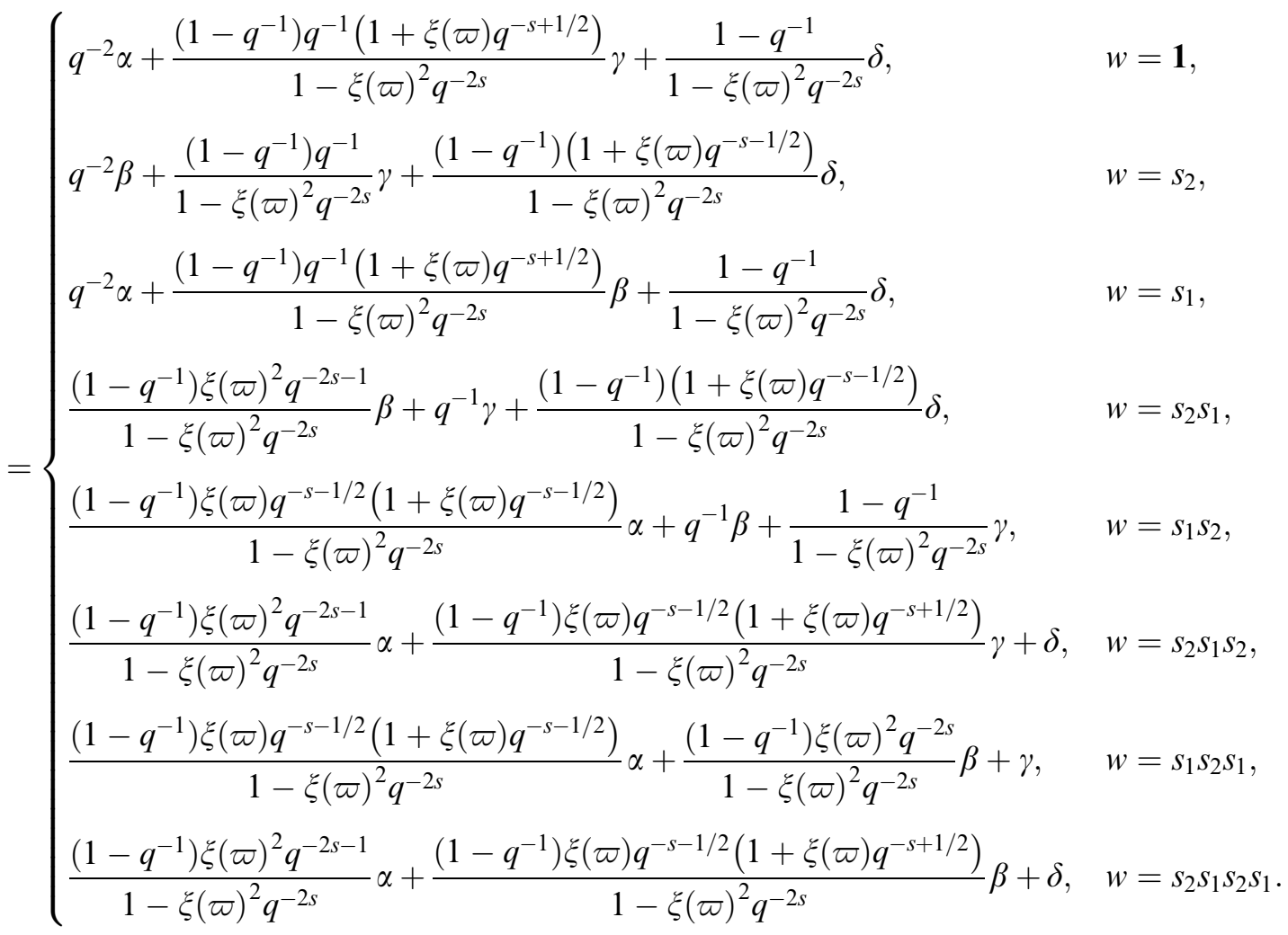

This holds for any unramified character $\xi$. 
Proof. If the conjugation $g \mapsto w^{-1} g w$ moves the $\mu$-variable to a positive root, we split the $\mu$-integral as $\int_{\mathfrak{D}}+\int_{F \backslash \mathfrak{D}}$, otherwise as $\int_{\mathfrak{p}}+\int_{F \backslash \mathfrak{p}}$. Then one uses

$$
\begin{aligned}
& \left(\begin{array}{llll}
1 & & & \\
& 1 & & \\
\mu & & 1 & \\
\kappa & \mu & & 1
\end{array}\right)=\left(\begin{array}{llll}
-\mu^{-1} & & -1 & \\
\mu^{-2} \kappa & -\mu^{-1} & & -1 \\
& & -\mu & \\
& & -\kappa & -\mu
\end{array}\right)\left(\begin{array}{cccc}
1 & & & \\
& 1 & & \\
& & 1 & \\
\kappa \mu^{-2} & & & 1
\end{array}\right)
\end{aligned}
$$

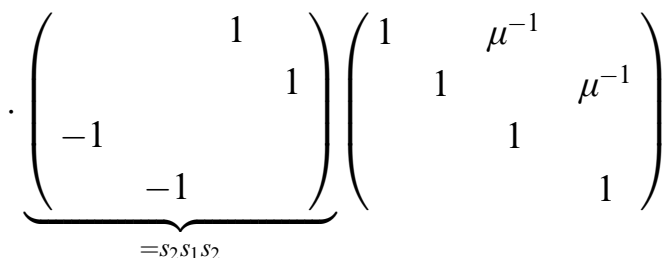

on the second integral and Lemma 2.2.

2.4. Lemma. With $f$ and $\xi$ as in the previous lemmas, we have

$$
\begin{aligned}
& (A(s) f)(w)=\int_{F^{3}} f\left(\left(\begin{array}{cccc}
1 & & & \\
& 1 & & \\
\mu & x & 1 & \\
\kappa & \mu & & 1
\end{array}\right) s_{2} s_{1} s_{2} w\right) d x d \mu d \kappa \\
& \begin{cases}\frac{\left(1-q^{-1}\right) \xi(\varpi)^{2} q^{-2 s}\left(1-\xi(\varpi) q^{-s-3 / 2}\right)}{\left(1-\xi(\varpi)^{2} q^{-2 s}\right)\left(1-\xi(\varpi) q^{-s+1 / 2}\right)} \alpha+\frac{\left(1-q^{-1}\right) \xi(\varpi) q^{-s-1 / 2}}{1-\xi(\varpi) q^{-s+1 / 2}} \beta & \\
\quad+\frac{\left(1-q^{-1}\right) \xi(\varpi) q^{-s+1 / 2}}{1-\xi(\varpi) q^{-s+1 / 2}} \gamma+\delta, & w=\mathbf{1}, \\
\frac{\left(1-q^{-1}\right) \xi(\varpi) q^{-s-3 / 2}}{1-\xi(\varpi) q^{-s+1 / 2}} \alpha+q^{-1} \gamma+\frac{\left(1-q^{-1}\right)}{1-\xi(\varpi) q^{-s+1 / 2}} \delta & \\
\quad+\frac{\left(1-q^{-1}\right) \xi(\varpi) q^{-s-1 / 2}\left(1-q^{-1}+\xi(\varpi) q^{-s+1 / 2}-\xi(\varpi)^{2} q^{-2 s}\right)}{\left(1-\xi(\varpi)^{2} q^{-2 s}\right)\left(1-\xi(\varpi) q^{-s+1 / 2}\right)} \beta, & w=s_{2}, \\
\frac{\left(1-q^{-1}\right) \xi(\varpi) q^{-s-3 / 2}}{1-\xi(\varpi) q^{-s+1 / 2}} \alpha q^{-2} \beta+\frac{\left(1-q^{-1}\right)}{1-\xi(\varpi) q^{-s+1 / 2}} \delta & \\
+\frac{\left(1-q^{-1}\right) q^{-1}\left(1-\xi(\varpi) q^{-s-1 / 2}+\xi(\varpi)^{2} q^{-2 s+1}-\xi(\varpi)^{2} q^{-2 s}\right)}{\left(1-\xi(\varpi)^{2} q^{-2 s}\right)\left(1-\xi(\varpi) q^{-s+1 / 2}\right)} \gamma, & w=s_{2} s_{1}, \\
q^{-3} \alpha+\frac{\left(1-q^{-1}\right) q^{-2}}{1-\xi(\varpi) q^{-s+1 / 2}} \beta+\frac{\left(1-q^{-1}\right) q^{-1}}{1-\xi(\varpi) q^{-s+1 / 2} \gamma} & \\
+\frac{\left(1-q^{-1}\right)\left(1-\xi(\varpi) q^{-s-3 / 2}\right)}{\left(1-\xi(\varpi)^{2} q^{-2 s}\right)\left(1-\xi(\varpi) q^{-s+1 / 2}\right)} \delta, & w=s_{2} s_{1} s_{2} .\end{cases}
\end{aligned}
$$

Proof. One has to split the $x$-integration and then use 


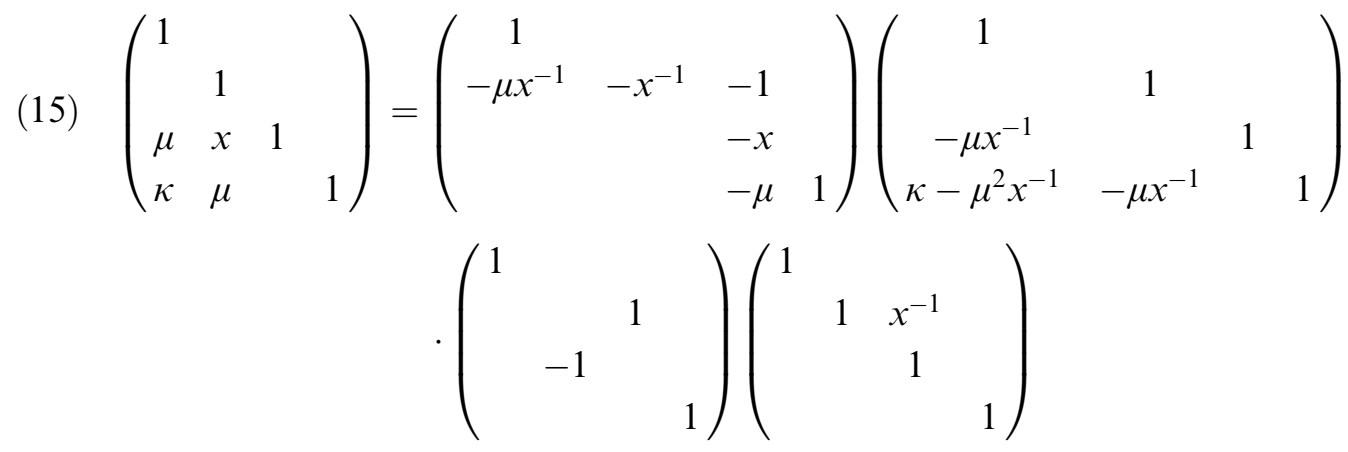

and Lemma 2.3.

2.5. Proposition. Let $V_{\xi, s}$ be the standard space of the induced representation $\xi v^{s} \mathbf{1}_{\mathrm{GL}(2)} \rtimes \xi^{-1} v^{-s}$.

(i) For each flat section $f_{s} \in V_{1, s}$ and each $g \in G(F)$, the limit

$$
\lim _{s \rightarrow-1 / 2}\left(A(s) f_{s}\right)(g)
$$

exists. This defines a non-zero intertwining operator $A(-1 / 2): V_{1,-1 / 2} \rightarrow V_{1,1 / 2}$. With respect to a suitable basis, the restriction of this operator to the four-dimensional spaces of I-invariant vectors has matrix

$$
\left(\begin{array}{cccc}
q^{-1} & -q^{-1} & -1 & 1 \\
-q^{-2} & q^{-2} & q^{-1} & -q^{-1} \\
-q^{-2} & q^{-2} & q^{-1} & -q^{-1} \\
q^{-3} & -q^{-3} & -q^{-2} & q^{-2}
\end{array}\right)
$$

(ii) Let $\xi$ be the non-trivial unramified quadratic character of $F^{*}$. For each flat section $f_{s} \in V_{\xi, s}$ and each $g \in G(F)$, the limit

$$
\lim _{s \rightarrow 1 / 2}\left(A(s) f_{s}\right)(g)
$$

exists. This defines a non-zero intertwining operator $A(1 / 2): V_{\xi, 1 / 2} \rightarrow V_{\xi,-1 / 2}$. With respect to a suitable basis, the restriction of this operator to the four-dimensional spaces of I-invariant vectors has matrix

$$
\left(\begin{array}{cccc}
\frac{1}{2}\left(1+q^{-2}\right) q^{-1} & -\frac{1}{2}\left(1-q^{-1}\right) q^{-1} & -\frac{1}{2}\left(1-q^{-1}\right) & 1 \\
-\frac{1}{2}\left(1-q^{-1}\right) q^{-2} & q^{-2} & q^{-1} & \frac{1}{2}\left(1-q^{-1}\right) \\
-\frac{1}{2}\left(1-q^{-1}\right) q^{-2} & q^{-2} & q^{-1} & \frac{1}{2}\left(1-q^{-1}\right) \\
q^{-3} & \frac{1}{2}\left(1-q^{-1}\right) q^{-2} & \frac{1}{2}\left(1-q^{-1}\right) q^{-1} & \frac{1}{2}\left(1+q^{-2}\right)
\end{array}\right)
$$


Proof. We prove (i), the argument for (ii) being very similar. From the formulas in Lemma 2.4 it is clear that the limit (16) exists for each $g$, provided $f_{s}$ is an $I$-invariant section. Let $V \subset V_{1,-1 / 2}$ be the $G$-invariant subspace generated by the $I$-invariant functions. Each $f \in V$ lies in a unique flat section $f_{s}$, and we can define

$$
(A(-1 / 2) f)(g):=\lim _{s \rightarrow-1 / 2}\left(A(s) f_{s}\right)(g) .
$$

The limit exists since $f$ is a linear combination of right translates of $I$-invariant functions, and $g$ in (16) is arbitrary. Taking the limit on

$$
\left(A(s) f_{s}\right)(g h)=\left(A(s) f_{s}^{h}\right)(g)
$$

shows that $A(-1 / 2)$ defines an intertwining operator $V \rightarrow V_{1, s}$. Now let $f_{1}, f_{2}, f_{3}, f_{4}$ be the basis of $V_{\xi,-1 / 2}^{I}$ such that the vectors $(\alpha, \beta, \gamma, \delta)$ as in (12) run through the standard unit vectors. With respect to this basis and the analogous one for $V_{1,1 / 2}^{I}$ it is easily computed from Proposition 2.4 that $A(-1 / 2)$ has the matrix given by (17). This matrix has rank 1. In particular, $A(-1 / 2)$ is neither zero nor injective, and so $V$ cannot be irreducible. Since we know the length of $v^{-1 / 2} \mathbf{1}_{\mathrm{GL}(2)} \rtimes v^{1 / 2}$ is 2 , it follows that $V$ is all of $V_{1,-1 / 2}$.

2.6. Corollary. (i) The representation $\Pi(\mathrm{St} \otimes \mathrm{St})=\tau\left(T, v^{-1 / 2}\right)$ has no non-zero $G(\mathfrak{o})$ fixed vector and, up to multiples, a unique non-zero I-invariant vector. If $\tau\left(T, v^{-1 / 2}\right)$ is realized as a subrepresentation of $v^{1 / 2} \mathbf{1}_{\mathrm{GL}(2)} \rtimes v^{-1 / 2}$, then the I-invariant function $f$ is given by the values

$$
\left(f(\mathbf{1}), f\left(s_{2}\right), f\left(s_{2} s_{1}\right), f\left(s_{2} s_{1} s_{2}\right)\right)=\left(1,-q^{-1},-q^{-1}, q^{-2}\right) .
$$

This function is even $P_{1}$-invariant (see the section on notations).

(ii) Let $\xi$ be the non-trivial unramified quadratic character of $F^{*}$. The representation $\Pi(\xi \mathrm{St} \otimes \mathbf{1})=L\left(\left(v^{1 / 2} \xi \mathrm{St}_{\mathrm{GL}(2)}, v^{-1 / 2}\right)\right)$ has no non-zero $G(\mathfrak{v})$-invariant vector, but has a twodimensional subspace of I-invariant vectors. The representation can be realized as a subrepresentation of $\xi v^{1 / 2} \mathbf{1}_{\mathrm{GL}(2)} \rtimes \xi v^{-1 / 2}$ in such a way that the space of I-invariant functions $f$ is spanned by the functions given by

$$
\left(f(\mathbf{1}), f\left(s_{2}\right), f\left(s_{2} s_{1}\right), f\left(s_{2} s_{1} s_{2}\right)\right)=\left(-(1+q) q^{2},(1-q) q,(1-q) q, 1+q\right)
$$

and

$$
\left(f(\mathbf{1}), f\left(s_{2}\right), f\left(s_{2} s_{1}\right), f\left(s_{2} s_{1} s_{2}\right)\right)=\left(-q^{2},-q^{2}, 1,1\right) .
$$

In fact, the function (21) is $P_{1}$-invariant, and the function (22) is $P_{2}$-invariant.

Proof. (i) From the previous proposition we get a non-zero and non-injective intertwining operator 


$$
A(-1 / 2): v^{-1 / 2} \mathbf{1}_{\mathrm{GL}(2)} \rtimes v^{1 / 2} \rightarrow v^{1 / 2} \mathbf{1}_{\mathrm{GL}(2)} \rtimes v^{-1 / 2} .
$$

On $I$-invariant vectors $A(s)$ has rank 1, by (17), thus one of the two constituents of $v^{-1 / 2} \mathbf{1}_{\mathrm{GL}(2)} \rtimes v^{1 / 2}$ contains a three-dimensional space of $I$-invariant vectors, the other one a one-dimensional space. The spherical vector in $V_{1,-1 / 2}$ has $(\alpha, \beta, \gamma, \delta)=(1,1,1,1)$ and lies in the kernel of $A(s)$. Therefore this kernel is isomorphic to $L\left(\left(v, \mathbf{1}_{F^{*}} \rtimes v^{-1 / 2}\right)\right)$, the spherical constituent of $v^{1 / 2} \mathbf{1}_{\mathrm{GL}(2)} \rtimes v^{-1 / 2}$; see [ST], Lemma 3.8. It follows that the image of $A(-1 / 2)$ in $V_{1,1 / 2}$ is isomorphic to $\tau\left(T, v^{-1 / 2}\right)$ and contains an essentially unique $I$ invariant vector. Its explicit form can be read off from (17). The function is obviously right-invariant under $s_{1}$ and therefore under $P_{1}$.

The argument for (ii) is very similar. The relevant matrix (19) has rank 2, its kernel is spanned by ${ }^{t}\left(-(1+q) q^{2},(1-q) q,(1-q) q, 1+q\right)$ and ${ }^{t}\left(-q^{2},-q^{2}, 1,1\right)$. The function (21) is right invariant under $s_{1}$ and therefore under $P_{1}$. The function $f$ as in (22) is right invariant under $s_{2}$ and therefore under $P_{2}$.

\section{Invariant vectors in $\Pi(\mathrm{St} \otimes \mathbf{1})$}

We shall now investigate the $p$-adic properties of the non-tempered representation $\Pi(\mathrm{St} \otimes \mathbf{1})=L\left(\left(v^{1 / 2} \mathrm{St}, v^{-1 / 2}\right)\right)$. By [ST], Lemma 3.8, it occurs as a subrepresentation of $\mathbf{1}_{F^{*}} \rtimes \mathbf{1}_{\mathrm{GL}(2)}$ (induction from the parabolic $Q$ ). We therefore consider the family of induced representations

$$
v^{s} \rtimes v^{-s / 2} \mathbf{1}_{\mathrm{GL}(2)}, \quad s \in \mathbb{C},
$$

and apply a similar method as in the previous section. Let $V_{s}$ be the standard model for $v^{s} \rtimes v^{-s / 2} \mathbf{1}_{\mathrm{GL}(2)}$, i.e., $V_{s}$ consists of locally constant functions $f: G(F) \rightarrow \mathbb{C}$ that transform as

$$
f\left(\left(\begin{array}{ccc}
u & * & * \\
& A & * \\
& & u^{-1} \operatorname{det}(A)
\end{array}\right) g\right)=|u|^{2+s}|\operatorname{det}(A)|^{-s / 2-1} f(g), \quad u \in F^{*}, A \in \operatorname{GL}(2, F)
$$

(the modular factor of the parabolic $Q$ is $\left|u^{4} \operatorname{det}(A)^{-2}\right|$ ). Let $V_{s}^{I}$ be the subspace of $I$ invariant vectors. Restricting functions in $V_{s}$ from $G(F)$ to $G(\mathfrak{p})$ is an injective operation, thus $V_{s}^{I}$ is isomorphic to the space of functions $G(\mathfrak{v}) \rightarrow \mathbb{C}$ that are left $Q(\mathfrak{p})$-invariant and right $I$-invariant. Now

$$
Q(\mathfrak{o}) \backslash G(\mathfrak{o}) / I \simeq Q(\mathfrak{o} / \mathfrak{p}) \backslash G(\mathfrak{o} / \mathfrak{p}) / B(\mathfrak{o} / \mathfrak{p}) .
$$

Since $s_{2} \in Q$, a complete set of representatives for this double coset space is $\left\{1, s_{1}, s_{1} s_{2}, s_{1} s_{2} s_{1}\right\}$. It follows that $\operatorname{dim}_{\mathbb{C}}\left(V_{s}^{I}\right)=4$; any $f \in V_{s}^{I}$ is determined by the values

$$
\alpha:=f(1), \quad \beta:=f\left(s_{1}\right), \quad \gamma:=f\left(s_{1} s_{2}\right), \quad \delta:=f\left(s_{1} s_{2} s_{1}\right),
$$


and these values can be chosen arbitrarily. We would like to determine, for $s=0$, the intersection of $V_{s}^{I}$ with a subspace $W_{0}$ of $V_{0}$ that carries the representation $L\left(\left(v^{1 / 2} \mathrm{St}, v^{-1 / 2}\right)\right)$. For this purpose we consider the intertwining operator $A(s): V_{s} \rightarrow V_{-s}$ defined by

$$
(A(s) f)(g)=\int_{H} f\left(w_{l} n g\right) d n, \quad w_{l}=s_{2} s_{1} s_{2} s_{1}=\left(\begin{array}{rrrr} 
& & & 1 \\
& & & \\
& -1 & &
\end{array}\right) .
$$

Here $H$ is the unipotent radical of the parabolic $Q$.

3.1. Proposition. The intertwining operator $A(s)$ defined by (24) is convergent for $\operatorname{Re}(s)>1$. It has meromorphic continuation to all of $\mathbb{C}$ with possible poles at points $s \in \mathbb{C}$ where $q^{-s \pm 1}=1$ or $q^{-2 s}=1$, and defines a non-zero intertwining map $v^{s} \rtimes v^{-s / 2} \mathbf{1}_{\mathrm{GL}(2)} \rightarrow v^{-s} \rtimes v^{s / 2} \mathbf{1}_{\mathrm{GL}(2)}$.

Proof. The proof is analogous to that of Proposition 2.1.

3.2. Lemma. With $f$ as in (23), we have for $\operatorname{Re}(s)>-1$ and $w \in W$

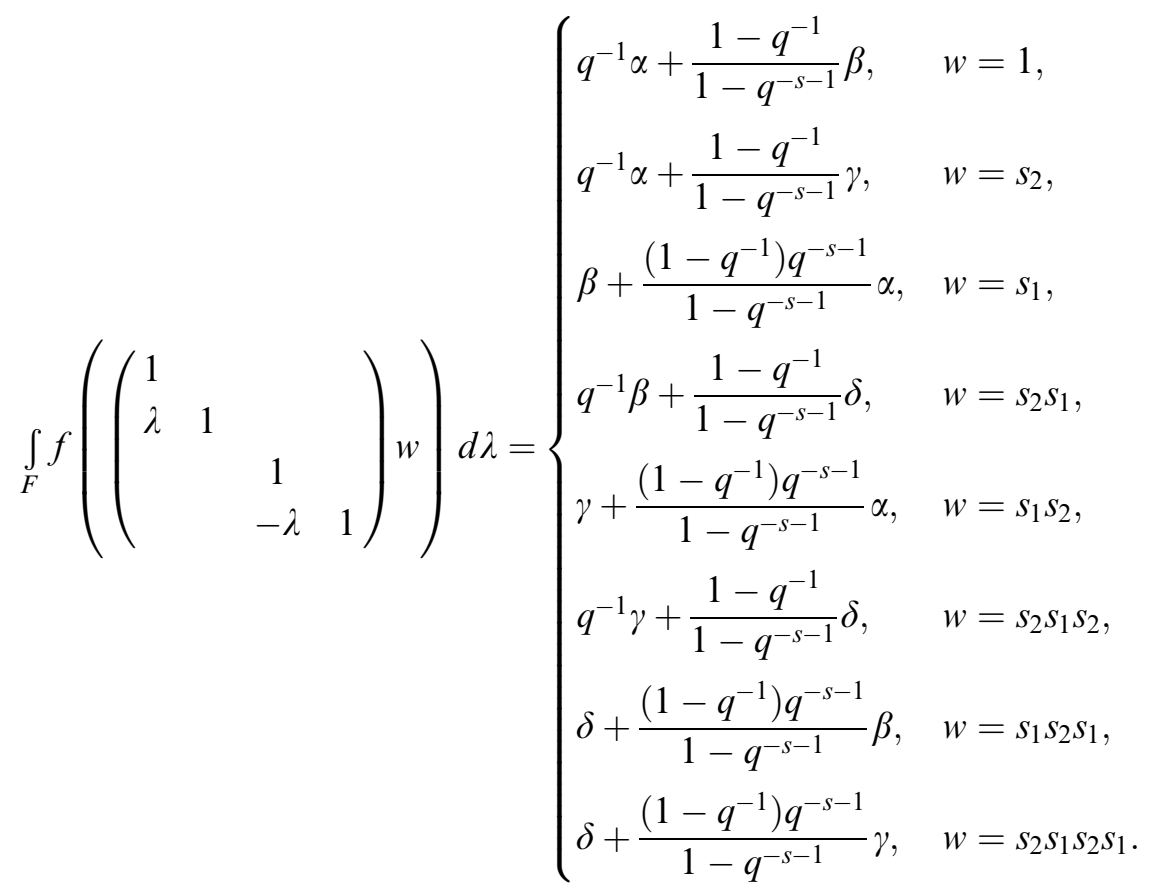

Proof. If the conjugation $g \mapsto w^{-1} g w$ moves the $\lambda$-variable to a positive root, we split the integral as $\int_{\mathfrak{D}}+\int_{F \backslash \mathfrak{D}}$, otherwise as $\int_{\mathfrak{p}}+\int_{F \backslash \mathfrak{p}}$. In either case the first integral is trivial, while the second one can be computed using (13).

3.3. Lemma. With $f$ as in (23), define 


$$
B(w):=\int_{F} f\left(\left(\begin{array}{cccc}
1 & & & \\
\lambda & 1 & & \\
& & 1 & \\
\kappa & & -\lambda & 1
\end{array}\right) w\right) d \lambda, \quad w \in W .
$$

Then, for $\operatorname{Re}(s)>0$,

$$
B(w)= \begin{cases}q^{-2} \alpha+\frac{\left(1-q^{-1}\right) q^{-1}}{1-q^{-s}} \beta+\frac{1-q^{-1}}{1-q^{-s}} \delta, & w=1, \\ q^{-2} \alpha+\frac{\left(1-q^{-1}\right) q^{-1}}{1-q^{-s}} \gamma+\frac{1-q^{-1}}{1-q^{-s}} \delta, & w=s_{2}, \\ \frac{\left(1-q^{-1}\right) q^{-s-1}}{1-q^{-s}} \alpha+q^{-1} \beta+\frac{1-q^{-1}}{1-q^{-s}} \gamma, & w=s_{1}, \\ \frac{\left(1-q^{-1}\right) q^{-s-1}}{1-q^{-s}} \alpha+\frac{\left(1-q^{-1}\right) q^{-s}}{1-q^{-s}} \beta+\gamma, & w=s_{1} s_{2}, \\ \frac{\left(1-q^{-1}\right) q^{-s-1}}{1-q^{-s}} \beta+q^{-1} \gamma+\frac{1-q^{-1}}{1-q^{-s}} \delta, & w=s_{2} s_{1} s_{2}, \\ \frac{\left(1-q^{-1}\right) q^{-s-1}}{1-q^{-s}} \alpha+\frac{\left(1-q^{-1}\right) q^{-s}}{1-q^{-s}} \beta+\delta, & w=s_{1} s_{2} s_{1}, \\ \frac{\left(1-q^{-1}\right) q^{-s-1}}{1-q^{-s}} \alpha+\frac{\left(1-q^{-1}\right) q^{-s}}{1-q^{-s}} \gamma+\delta, & w=s_{2} s_{1} s_{2} s_{1} .\end{cases}
$$

Proof. If the conjugation $g \mapsto w^{-1} g w$ moves the $\kappa$-variable to a positive root, we split the $\kappa$-integration as $\int_{\mathfrak{D}}+\int_{F \backslash \mathfrak{v}}$, otherwise as $\int_{\mathfrak{p}}+\int_{F \backslash \mathfrak{p}}$. We then use the formula

$$
\begin{aligned}
& \left(\begin{array}{cccc}
1 & & & \\
\lambda & 1 & & \\
& & 1 & \\
\kappa & & -\lambda & 1
\end{array}\right)=\left(\begin{array}{ccccc}
\kappa^{-1} & & \kappa^{-1} \lambda & 1 \\
& 1 & \kappa^{-1} \lambda^{2} & \lambda \\
& & 1 & \\
& & & \kappa
\end{array}\right)\left(\begin{array}{ccccc}
1 & & & \\
\kappa^{-1} \lambda & 1 & & \\
& & 1 & \\
& & -\kappa^{-1} \lambda & 1
\end{array}\right) \\
& \underbrace{\left(\begin{array}{cccc} 
& & & \\
& 1 & & \\
& & 1 & \\
-1 & & &
\end{array}\right)}_{=s_{1} S_{2} s_{1}}\left(\begin{array}{cccc}
-1 & & & -\kappa^{-1} \\
& 1 & & \\
& & 1 & \\
& & & -1
\end{array}\right)
\end{aligned}
$$

on the second integral and Lemma 3.2.

The intertwining operator $A(s)$ induces a linear map between the four-dimensional spaces $V_{s}^{I}$ and $V_{-s}^{I}$. Using Lemma 3.3, we can now explicitly compute this linear map. 
3.4. Lemma.

$$
(A(s) f)(w)= \begin{cases}\frac{\left(1-q^{-1}\right) q^{-s-1}}{1-q^{-s+1}} \alpha+\frac{\left(1-q^{-1}\right) q^{-s}}{1-q^{-s+1}} \beta+\frac{\left(1-q^{-1}\right) q^{-s+1}}{1-q^{-s+1}} \gamma+\delta, & w=1, \\ \frac{\left(1-q^{-1}\right) q^{-s-1}}{1-q^{-s+1}} \alpha+\frac{\left(1-q^{-1}\right) q^{-s}}{1-q^{-s+1}} \beta+q^{-1} \gamma+\frac{1-q^{-1}}{1-q^{-s+1}} \delta, & w=s_{1}, \\ \frac{\left(1-q^{-1}\right) q^{-s-1}}{1-q^{-s+1}} \alpha+q^{-2} \beta+\frac{\left(1-q^{-1}\right) q^{-1}}{1-q^{-s+1}} \gamma+\frac{1-q^{-1}}{1-q^{-s+1}} \delta, & w=s_{1} s_{1}, \\ q^{-3} \alpha+\frac{\left(1-q^{-1}\right) q^{-2}}{1-q^{-s+1}} \beta+\frac{\left(1-q^{-1}\right) q^{-1}}{1-q^{-s+1}} \gamma+\frac{1-q^{-1}}{1-q^{-s+1}} \delta, & w=s_{1} s_{2} s_{1} .\end{cases}
$$

Proof. One uses the matrix identity

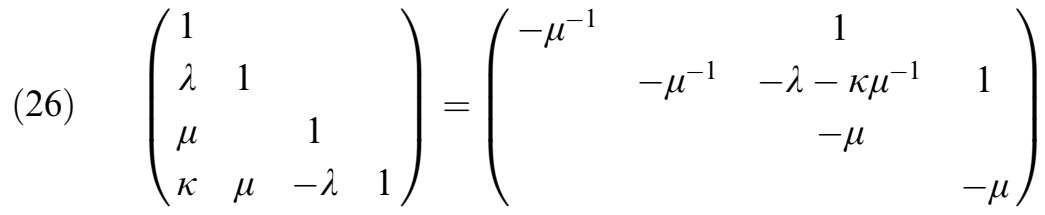

$$
\begin{aligned}
& \cdot\left(\begin{array}{cccc}
1 & & & \\
-\kappa \mu^{-1} & 1 & & \\
-\mu^{-2} \kappa+\mu^{-1} \lambda & & 1 & \\
\kappa \mu^{-1} & 1
\end{array}\right) \underbrace{\left(\begin{array}{lll} 
& 1 & \\
& & 1 \\
-1 & & \\
& -1
\end{array}\right)}_{=S_{2} S_{1} S_{2}} \\
& \left(\begin{array}{cccc}
1 & & -\mu^{-1} & \\
& 1 & & -\mu^{-1} \\
& & 1 & \\
& & & 1
\end{array}\right)
\end{aligned}
$$

and the results of the previous lemma. limit

3.5. Proposition. For each flat section $f_{s} \in v^{s} \mathbf{1}_{\mathrm{GL}(2)} \rtimes v^{-s}$ and each $g \in G(F)$, the

$$
\lim _{s \rightarrow 0}\left(A(s) f_{s}\right)(g)
$$

exists. This defines a non-zero intertwining operator

$$
A(0): \mathbf{1}_{F^{*}} \rtimes \mathbf{1}_{\mathrm{GL}(2)} \rightarrow \mathbf{1}_{F^{*}} \rtimes \mathbf{1}_{\mathrm{GL}(2)} .
$$

With respect to a suitable basis, the restriction of this operator to the four-dimensional space of I-invariant vectors has matrix

$$
\left(\begin{array}{cccc}
-q^{-2} & -q^{-1} & -1 & 1 \\
-q^{-2} & -q^{-1} & q^{-1} & -q^{-1} \\
-q^{-2} & q^{-2} & -q^{-2} & -q^{-1} \\
q^{-3} & -q^{-3} & -q^{-2} & -q^{-1}
\end{array}\right)
$$


Proof. We argue as in Proposition 2.5. It follows from the formulas in Lemma 3.4 that the limit (27) exists for each $g$, provided $f_{s}$ is an $I$-invariant section. Let $W \subset V_{0}$ be the $G$-invariant subspace generated by the $I$-invariant functions. Each $f \in W$ lies in a unique flat section $f_{s}$, and we can define

$$
(A(0) f)(g):=\lim _{s \rightarrow 0}\left(A(s) f_{s}\right)(g) .
$$

The limit exists since $f$ is a linear combination of right translates of $I$-invariant functions, and $g$ in (27) is arbitrary. This defines an intertwining operator $A(0): W \rightarrow V_{0}$. Now consider the basis of $V_{0}^{I}$ consisting of those four functions $f$ such that $(\alpha, \beta, \gamma, \delta)$ as defined in (23) are the standard unit vectors. With respect to this basis it is easily computed from Lemma 3.4 that the endomorphism of $V_{0}^{I}$ induced by $A(0)$ has the matrix given by (28). This matrix is invertible, with characteristic polynomial

$$
\chi_{A(0)}(X)=\left(X+q^{-2}+q^{-1}\right)^{3}\left(X-q^{-2}-q^{-1}\right) .
$$

It follows that the intertwining operator

$$
A(0)-\left(q^{-2}+q^{-1}\right) \operatorname{id}_{V_{0}}: W \rightarrow V_{0}
$$

is neither zero nor injective, and so $W$ cannot be irreducible. Since we know the length of $\mathbf{1}_{F^{*}} \rtimes \mathbf{1}_{\mathrm{GL}(2)}$ is 2 , it follows that $W=V_{0}$.

3.6. Corollary. The representation $\Pi(\mathrm{St} \otimes \mathbf{1})=L\left(\left(v^{1 / 2} \mathrm{St}, v^{-1 / 2}\right)\right)$ has no non-zero $P_{1}$-invariant vector, but a non-zero I-invariant vector, unique up to multiples. If the representation is realized as a subrepresentation of $\mathbf{1}_{F^{*}} \rtimes \mathbf{1}_{\mathrm{GL}(2)}$, then the I-invariant function $f$ is given by the values

$$
\left(f(\mathbf{1}), f\left(s_{1}\right), f\left(s_{1} s_{2}\right), f\left(s_{1} s_{2} s_{1}\right)\right)=\left(1,-q^{-1},-q^{-1}, q^{-2}\right) .
$$

This function is even invariant under the Klingen congruence subgroup $P_{2}$.

Proof. From Proposition 3.5 we get a non-zero and non-injective intertwining operator

$$
\varphi:=A(0)-\left(q^{-2}+q^{-1}\right) \mathrm{id}_{V_{0}}: \mathbf{1}_{F^{*}} \rtimes \mathbf{1}_{\mathrm{GL}(2)} \rightarrow \mathbf{1}_{F^{*}} \rtimes \mathbf{1}_{\mathrm{GL}(2)} .
$$

Let $W \subset V_{0}$ be the kernel of $\varphi$. It follows from (28) that $W$ contains a unique $I$-invariant function (up to scalars), namely the one given by (29). Since $s_{1} \in P_{1}$, this function is not $P_{1}$-invariant. It is therefore also not $G(\mathfrak{v})$-invariant. Since the unique spherical constituent of $\mathbf{1}_{F^{*}} \rtimes \mathbf{1}_{\mathrm{GL}(2)}$ is $L\left(\left(v, \mathbf{1}_{F^{*}} \rtimes v^{-1 / 2}\right)\right)$ (see [ST], Lemma 3.8), it follows that $W$ carries the representation $L\left(\left(v^{1 / 2} \mathrm{St}, v^{-1 / 2}\right)\right)$. The vector (29) is $P_{2}$-invariant since it is obviously $s_{2}$-invariant.

\section{Atkin-Lehner involutions and $\varepsilon$-factors}

We consider the Atkin-Lehner element $\eta$ defined in (6). Since $\eta$ normalizes the Iwahori subgroup $I$, the operator $\pi(\eta)$ acts on the space of $I$-invariant vectors, for any repre- 
sentation $\pi$ of $\operatorname{GSp}(4, F)$. If $\pi$ has trivial central character, then $\pi(\eta)$ acts as an involution, because $\eta^{2}=\varpi \mathbf{1}$. We call these operators Atkin-Lehner involutions. They split the spaces of $I$-invariant vectors into \pm 1 -eigenspaces. If $v$ is an eigenvector, we call its eigenvalue the Atkin-Lehner eigenvalue of $v$. The Atkin-Lehner involutions also act on the space of $P_{1}$ invariant vectors and on the space of $P_{02}$-invariant vectors, since these parahoric subgroups are also normalized by $\eta$.

Consider the representation $\Pi=\Pi(\mathrm{St} \otimes \mathrm{St})=\tau\left(T, v^{-1 / 2}\right)$ with its one-dimensional space of $I$-invariant vectors given explicitly in Corollary 2.6 (i). Let $f$ be the $P_{1}$-invariant function given by

$$
\left(f(\mathbf{1}), f\left(s_{2}\right), f\left(s_{2} s_{1}\right), f\left(s_{2} s_{1} s_{2}\right)\right)=\left(1,-q^{-1},-q^{-1}, q^{-2}\right)
$$

in the standard induced model of $v^{1 / 2} \mathbf{1}_{\mathrm{GL}(2)} \rtimes v^{-1 / 2}$. The eigenvalue of $\Pi(\eta)$ on $f$ is very easily computed: We have

$$
(\Pi(\eta) f)(\mathbf{1})=f(\eta)=\left|-\varpi^{-1}\right|^{2} f\left(s_{2} s_{1} s_{2}\right)=1=f(\mathbf{1})
$$

and similarly $(\Pi(\eta) f)(w)=f(w)$ for $w \in\left\{s_{2}, s_{2} s_{1}, s_{2} s_{1} s_{2}\right\}$. Thus $\Pi(\eta) f=f$, and the Atkin-Lehner eigenvalue of $f$ is 1 .

Next consider the representation $\Pi=\Pi(\xi \mathrm{St} \otimes \mathbf{1})=L\left(\left(v^{1 / 2} \xi \mathrm{St}_{\mathrm{GL}(2)}, v^{-1 / 2}\right)\right)$, where $\xi$ is the unramified quadratic character of $F^{*}$. According to Corollary 2.6 (ii), we now have a two-dimensional space of $I$-invariant vectors. A computation very similar to the one above shows that

$$
\Pi(\eta) f=f \quad \text { for each } I \text {-invariant } f \in \Pi(\xi \mathrm{St} \otimes \mathbf{1}) \subset \xi v^{1 / 2} \mathbf{1}_{\mathrm{GL}(2)} \rtimes \xi v^{-1 / 2} .
$$

In other words, the Atkin-Lehner eigenvalue is 1 on the whole space of $I$-invariant vectors. This also implies that the vector $(22)$ is not only invariant under $P_{2}$ but even under the paramodular group $P_{02}$.

Finally consider the representation $\Pi=\Pi(\mathrm{St} \otimes \mathbf{1})=L\left(\left(v^{1 / 2} \mathrm{St}, v^{-1 / 2}\right)\right)$. According to Corollary 3.6 there is an essentially unique $I$-invariant function

$$
f \in \Pi(\mathrm{St} \otimes \mathbf{1}) \subset \mathbf{1}_{F^{*}} \rtimes \mathbf{1}_{\mathrm{GL}(2)}
$$

given by

$$
\left(f(\mathbf{1}), f\left(s_{1}\right), f\left(s_{1} s_{2}\right), f\left(s_{1} s_{2} s_{1}\right)\right)=\left(1,-q^{-1},-q^{-1}, q^{-2}\right) .
$$

A similar computation as before yields $\Pi(\eta) f=-f$, i.e., the Atkin-Lehner eigenvalue of $f$ is -1 . Moreover it shows that the above vector is not only invariant under $P_{2}$ but even under $P_{02}$.

The following table gives, for each of the three representations we investigated above, the dimensions of the spaces of vectors invariant under the subgroups indicated in the top row. These are the results of sections 2 and 3. The next-to-last column gives the AtkinLehner eigenvalues we just computed. 


\begin{tabular}{|l|c|c|c|c|c|c|}
\hline representation & $I$ & $P_{1}$ & $P_{2}$ & $P_{02}$ & AL-eigenvalue & $\varepsilon(1 / 2, \Pi)$ \\
\hline \hline$\Pi(\mathrm{St} \otimes \mathbf{1})$ & 1 & 0 & 1 & 1 & -1 & -1 \\
\hline$\Pi(\xi \mathrm{St} \otimes \mathbf{1})$ & 2 & 1 & 1 & 1 & 1 & 1 \\
\hline$\Pi(\mathrm{St} \otimes \mathrm{St})$ & 1 & 1 & 0 & 0 & 1 & 1 \\
\hline
\end{tabular}

We have noted above that under some reasonable assumptions on the local Langlands correspondence, the representation $\Pi(\mathrm{St} \otimes \mathbf{1})$ is a functorial lift of the representation $\mathrm{St} \otimes 1$ of $\operatorname{PGL}(2, F) \times \operatorname{PGL}(2, F)$, and similarly for the other two representations. Therefore the $\varepsilon$-factor of $\Pi\left(\pi_{1} \otimes \pi_{2}\right)$ should be given by $\varepsilon\left(s, \pi_{1}, \psi\right) \varepsilon\left(s, \pi_{2}, \psi\right)$. Noting that

$$
\varepsilon(1 / 2, \mathrm{St})=-1, \quad \varepsilon(1 / 2, \xi \mathrm{St})=1, \quad \varepsilon(1 / 2, \mathbf{1})=1
$$

we have listed the value of $\varepsilon\left(1 / 2, \pi_{1}\right) \varepsilon\left(1 / 2, \pi_{2}\right)$ in the last column of table (30). Note that these values do not depend on the choice of $\psi$. We see that all our Atkin-Lehner eigenvalues coincide with the signs defined by $\varepsilon$-factors. Hence the situation is similar as for PGL(2), where the sign defined by the $\varepsilon$-factor always coincides with the Atkin-Lehner eigenvalue on the local newform; see [Sch1].

\section{Classical modular forms}

In this final chapter our global ground field is $F=\mathbb{Q}$. We shall use the main lifting result, Theorem 1.1, to construct certain holomorphic Siegel modular forms of degree 2 . The congruence properties of these modular forms will be controlled by our local results as summarized in (30). In the classical theory of Siegel modular forms it is customary to realize symplectic groups using the symplectic form

$$
J=\left(\begin{array}{rr}
\mathbf{1}_{2} \\
-\mathbf{1}_{2} &
\end{array}\right)
$$

Thus, we change our notation from now on and define, using this $J$,

$$
G=\mathrm{GSp}(4)=\left\{g \in \mathrm{GL}(4):{ }^{t} g J g=\lambda(g) J \text { for some } \lambda(g) \in \mathrm{GL}(1)\right\}
$$

and similarly for $\mathrm{Sp}(4)$. An isomorphism between this $\mathrm{GSp}(4)$ and our previous version is given by switching the first two rows and the first two columns. Siegel modular forms of degree 2 are holomorphic functions on the Siegel upper half space

$$
\mathbb{H}_{2}=\{M \in M(2, \mathbb{C}) \text { symmetric, } \operatorname{Im}(M) \text { positive definite }\}
$$

with certain invariance properties. We refer to $[\mathrm{Fr}]$ for the precise definition (we shall allow modular forms for arbitrary arithmetic subgroups of $\operatorname{Sp}(4, \mathbb{Q}))$. Note that $G \operatorname{Sp}(4, \mathbb{R})^{+}$, the index-2 subgroup of $\operatorname{GSp}(4, \mathbb{R})$ of elements with positive multiplier, acts on $\mathbb{H}_{2}$ by the usual linear fractional transformations,

$$
Z \mapsto g\langle Z\rangle:=(A Z+B)(C Z+D)^{-1}, \quad g=\left(\begin{array}{cc}
A & B \\
C & D
\end{array}\right) \in \mathrm{GSp}(4, \mathbb{R})^{+}
$$


We shall also use the classical notation

$$
j(g, Z)=\operatorname{det}(C Z+D) \quad \text { for } Z \in \mathbb{H}_{2} \text { and } g=\left(\begin{array}{cc}
A & B \\
C & D
\end{array}\right) \in \mathrm{GSp}(4, \mathbb{R})^{+} \text {. }
$$

We shall now describe how to extract a classical modular form from an automorphic representation of $\operatorname{PGSp}(4, \mathbb{A})$; see also [AS] and [Yo3]. Assume that $\Pi=\otimes \Pi_{p}$ is the decomposition into local components of a cuspidal automorphic representation of $\operatorname{PGSp}(4, \mathbb{A})$. Assume that the archimedean component $\Pi_{\infty}$ is $\sigma_{k}^{+}$, the holomorphic discrete series representation of $\operatorname{PGSp}(4, \mathbb{R})$ with scalar minimal $K$-type $(k, k)$ (see [Sch2], section 4$)$. It is these representations that underlie holomorphic Siegel modular forms of weight $k$; see [AS]. Note that $\Pi_{\infty}$ contains a distinguished lowest weight vector $\Phi_{\infty}$ of weight $(k, k)$.

For each finite $p$ let $K_{p}$ be an open compact subgroup of $G\left(\mathbb{Q}_{p}\right)$ such that $K_{p}=G\left(\mathbb{Z}_{p}\right)$ for almost all $p$. Let $\Phi_{p} \in \Pi_{p}$ be a non-zero vector fixed by $K_{p}$. We also require that $\Phi_{p}$ is for almost all $p$ the distinguished vector used to define the restricted tensor product $\otimes \Pi_{p}$. All the local vectors can then be pieced together to define an element

$$
\Phi:=\bigotimes \Phi_{p} \in \Pi
$$

The representation $\Pi$ is realized as a space of automorphic forms, so we consider $\Phi$ a function on $G(\mathbb{A})$. By definition this function has the property

$$
\Phi\left(\rho g h_{\infty} h_{f}\right)=j\left(h_{\infty}, I\right)^{-k} \Phi(g) \text { for all } \rho \in G(\mathbb{Q}), g \in G(\mathbb{A}), h_{\infty} \in K_{\infty}, h_{f} \in K_{f}
$$

Here $K_{f}=\prod_{p<\infty} K_{p}$, and

$$
K_{\infty}=\left\{\left(\begin{array}{cc}
A & B \\
-B & A
\end{array}\right) \in \mathrm{GL}(4, \mathbb{R}): A^{t} A+B^{t} B=\mathbf{1}, A^{t} B=B^{t} A\right\} \simeq U(2)
$$

is the standard maximal compact subgroup of $\operatorname{Sp}(4, \mathbb{R})$. Note that $h_{\infty} \mapsto j\left(h_{\infty}, I\right)$, where $I=\left(\begin{array}{ll}i & \\ & i\end{array}\right)$, is a character of $K_{\infty}$.

5.1. Lemma. Let $G=\operatorname{GSp}(2 n)$. For each prime number $p$ let $K_{p}$ be an open compact subgroup of $G\left(\mathbb{Q}_{p}\right)$ such that $K_{p}=G\left(\mathbb{Z}_{p}\right)$ for almost all $p$, and such that the multiplier map $K_{p} \rightarrow \mathbb{Z}_{p}^{*}$ is surjective for all $p$. Then

$$
G(\mathbb{A})=G(\mathbb{Q}) G(\mathbb{R})^{+} K_{f}, \quad K_{f}=\prod_{p<\infty} K_{p},
$$

where $G(\mathbb{R})^{+}$is the group of elements of $G(\mathbb{R})$ with positive multiplier.

This lemma is well known and not hard to prove, making use of strong approximation for the simply connected algebraic group $\operatorname{Sp}(2 n)$. Together with equation (31) it implies that $\Phi$ as above is determined by its values on $G(\mathbb{R})^{+}$, provided the local compact subgroups are "big enough", as we shall assume from now on. Let us define a function $F$ on $\mathbb{H}_{2}$ by putting 


$$
F(Z)=\lambda\left(g_{\infty}\right)^{-k} j\left(g_{\infty}, I\right)^{k} \Phi\left(g_{\infty}\right), \quad \text { where } g_{\infty} \in G(\mathbb{R})^{+} \text {is such that } g_{\infty}\langle I\rangle=Z
$$

The stabilizer of $I$ in $G(\mathbb{R})^{+}$is $K_{\infty}$ times the center, so $F$ is well-defined by (31) and because of the factor $\lambda\left(g_{\infty}\right)^{-k}$. The fact that $\Phi_{\infty}$ is a lowest weight vector for the discrete series representation $\sigma_{k}^{+}$implies that $F$ is a holomorphic function; see [AS], 4.2. If we define the congruence subgroup

$$
\Gamma:=G(\mathbb{Q}) \cap G(\mathbb{R})^{+} \prod_{p<\infty} K_{p} \subset \operatorname{Sp}(4, \mathbb{Q}),
$$

then the function $F$ has the invariance property

$$
F(Z)=(F \mid \gamma)(Z):=j(\gamma, Z)^{-k} F(\gamma\langle Z\rangle) \text { for all } \gamma \in \Gamma \text {. }
$$

In other words, $F$ is a Siegel modular form of weight $k$ with respect to the congruence subgroup $\Gamma$. One can prove that the fact that the representation $\Pi$ is cuspidal implies that $F$ is a cusp form in the classical sense, meaning that only positive definite matrices occur in the Fourier expansion of $F$ and all $F \mid \rho, \rho \in \mathrm{Sp}(4, \mathbb{Q})$.

Let $f \in S_{2 k-2}\left(\Gamma_{0}(m)\right)$ be a classical elliptic holomorphic cusp form of weight $2 k-2$ and level $m$. Assuming that $f$ is an eigenform for all Hecke operators $T(p)$ with $p \nmid m$, there is a well-known procedure to attach a cuspidal automorphic representation $\pi_{f}$ of $\operatorname{PGL}(2, \mathbb{A})$ to $f$, see $[\mathrm{Ge}], \S 5$ or $[\mathrm{Bu}], 3.6$. Because of the strong multiplicity one theorem for $\mathrm{GL}(2)$, the space of automorphic forms realizing $\pi_{f}$ inside $L_{0}^{2}\left(\mathrm{GL}(2, \mathbb{Q}) \backslash \mathrm{GL}(2, \mathbb{A}), \mathbf{1}_{\mathbb{A}^{*}}\right)$ is unique; here $\mathbf{1}_{\mathbb{A}^{*}}$ is the trivial character of $\mathbb{A}^{*} \simeq Z(\operatorname{GL}(2, \mathbb{A}))$. In the following we shall moreover assume that $f$ is a newform, because oldforms do not lead to any additional automorphic representations.

Now let us assume that a set $S$ of places of $\mathbb{Q}$ exists that satisfies the hypotheses of our lifting theorem 1.1, where we put $\pi=\pi_{f}$. Let us also assume that $\infty \in S$. Such an $S$ can always be found if $\pi_{f}=\bigotimes \pi_{f, p}$ with $\pi_{f, p}$ being square integrable for some finite $p$. Given such an admissible $S$, let $\Pi=\Pi\left(\pi \otimes \pi_{S}\right)$ be the global lifting whose existence is guaranteed by Theorem 1.1. By that theorem, it is a cuspidal automorphic representation of $\operatorname{PGSp}(4, \mathbb{A})$. Let $\Pi=\bigotimes_{p \leqq \infty} \Pi_{p}$ be the factorization of $\Pi$ into local components.

Our modular form $f$ has weight $2 k-2$, and this determines the archimedean component of $\pi_{f}$, namely, $\pi_{f, \infty}=\mathscr{D}(2 k-3)$. Since we have assumed $\infty \in S$, the local archimedean lifting is

$$
\Pi_{\infty}=\Pi\left(\pi_{f, \infty} \otimes \mathrm{St}\right)=\Pi(\mathscr{D}(2 k-3) \otimes \mathscr{D}(1))=\sigma_{k}^{+}
$$

in the notation of [Sch2]. Recall that $\sigma_{k}^{+}$is the holomorphic discrete series representation of $\operatorname{PGSp}(4, \mathbb{R})$ with scalar minimal $K$-type $(k, k)$. As above let $\Phi_{\infty}$ be the distinguished lowest weight vector.

As for the finite places, we choose vectors $\Phi_{p} \in \Pi_{p}$, fixed under compact open subgroups $K_{p} \subset G\left(\mathbb{Q}_{p}\right)$, that allow us to define the global element $\Phi=\bigotimes \Phi_{p} \in \Pi$. As described above, $\Phi$ corresponds to a classical holomophic cuspform $F$ with respect to the congruence subgroup $\Gamma=G(\mathbb{Q}) \cap G(\mathbb{R})^{+} \prod_{p<\infty} K_{p} \subset \operatorname{Sp}(4, \mathbb{Q})$. 
We would like to control the level of these lifts, which of course depends on the local subgroups $K_{p}$. If $p \nmid m$, then $\pi_{f, p}$ as well as $\Pi_{p}$ are unramified representations, and we can choose $K_{p}=G\left(\mathbb{Z}_{p}\right)$ and $\Phi_{p}$ a spherical vector. These places do not impose any congruence conditions on $\gamma \in \Gamma$ (except for being $p$-integral). To control the level at the bad primes, we shall now assume that $m$ is square-free. Then we have

$$
\pi_{f, p}=\mathrm{St}_{\mathrm{GL}(2)} \quad \text { or } \quad \pi_{f, p}=\xi \mathrm{St}_{\mathrm{GL}(2)},
$$

where $\xi$ is the non-trivial unramified quadratic character of $\mathbb{Q}_{p}^{*}$. Which of the two representations occurs is decided by the eigenvalue of $f$ under the Atkin-Lehner involution (defined completely in classical terms, as in [Mi], 4.6, for example). If this eigenvalue is -1 , then $\pi_{f, p}=\mathrm{St}$, otherwise $\pi_{f, p}=\xi \mathrm{St}$.

In the following theorem we shall be talking about the $L$-function of a classical Siegel modular form $F$ (of degree 2). It is not clear whether this is a well-defined notion for eigenforms on arbitrary congruence subgroups. We therefore agree to associate an $L$-function to $F$ only if the following holds: $F$ is related to an adelic function $\Phi$ by formula (32); the $G(\mathbb{A})$-invariant subspace of $L_{0}^{2}\left(G(\mathbb{Q}) \backslash G(\mathbb{A}), \mathbf{1}_{\mathbb{A}^{*}}\right)$ generated by $\Phi$ carries a multiple of a cuspidal automorphic representation $\Pi$ of $G(\mathbb{A})$; and we know the local Langlands parameter for each local component of $\Pi$, so that we can define the spin (degree 4) $L$-function $L(s, \Pi)$. Under these circumstances, we define $L(s, F):=L(s, \Pi)$. If $F$ is a modular form for the full modular group and an eigenform for all Hecke operators, this definition of $L(s, F)$ coincides (up to a shift in the argument) with the usual spin $L$-function of $F$, see [AS]. In our square-free situation the only problematic local representations will be the three liftings

\section{$\Pi(\mathrm{St} \otimes \mathbf{1}), \quad \Pi(\xi \mathrm{St} \otimes \mathbf{1}), \quad \Pi(\mathrm{St} \otimes \mathrm{St})$}

we investigated before. All three representations are Iwahori-spherical, so we will define their local parameters to be those given by $[\mathrm{KL}]$. One can show that the local parameter of $\Pi(\mathrm{St} \otimes \mathbf{1})$ is then the direct sum of the local parameters for $\mathrm{St}_{\mathrm{GL}(2)}$ and $\mathbf{1}_{\mathrm{GL}(2)}$, and similarly for the other representations. The local $L$-factors of these representations are therefore given as follows.

\begin{tabular}{|c|c|}
\hline$\Pi_{p}$ & $L_{p}\left(s, \Pi_{p}\right)^{-1}$ \\
\hline \hline$\Pi(\mathrm{St} \otimes \mathbf{1})$ & $\left(1-p^{-s-1 / 2}\right)^{2}\left(1-p^{-s+1 / 2}\right)$ \\
\hline$\Pi(\xi \mathrm{St} \otimes \mathbf{1})$ & $\left(1-p^{-s-1 / 2}\right)\left(1-p^{-s+1 / 2}\right)\left(1+p^{-s-1 / 2}\right)$ \\
\hline$\Pi(\mathrm{St} \otimes \mathrm{St})$ & $\left(1-p^{-s-1 / 2}\right)^{2}$ \\
\hline
\end{tabular}

In the following theorem the paramodular group $\Gamma^{\text {para }}(m)$ is as in (3). The group $\Gamma_{0}(m)$ is the usual Hecke subgroup, defined by a congruence condition on the lower left block. We shall use the same symbol for subgroups of $\operatorname{SL}(2, \mathbb{Z})$ and of $\operatorname{Sp}(4, \mathbb{Z})$, hoping this causes no confusion.

5.2. Theorem. Let $m$ be a square-free positive integer and $f \in S_{2 k-2}\left(\Gamma_{0}(m)\right)$ an elliptic eigenform, assumed to be a newform. Let $\varepsilon_{p}$ be the eigenvalue of $f$ of the Atkin-Lehner involution at $p$. Let $\eta_{p}$ be the Atkin-Lehner involution in degree 2 at $p$. 
(i) If the sign in the functional equation of $L(s, f)$ is -1 , then there exists a cusp form $F \in S_{k}\left(\Gamma^{\mathrm{para}}(m)\right)$ of degree 2, unique up to multiples, whose completed spin L-function is given by

$$
L(s, F)=\frac{1}{4 \pi}\left(s-\frac{1}{2}\right) Z\left(s+\frac{1}{2}\right) Z\left(s-\frac{1}{2}\right) L(s, f),
$$

where $Z$ is the completed Riemann zeta function. This lifting preserves Atkin-Lehner eigenvalues, i.e., $\eta_{p} F=\varepsilon_{p} F$ for each $p$.

(ii) If $k$ is even, then there exists a cusp form $F \in S_{k}\left(\Gamma_{0}(m)\right)$ of degree 2 , unique up to multiples, whose completed spin L-function is given by

$$
L(s, F)=\frac{1}{4 \pi}\left(s-\frac{1}{2}\right)\left(\prod_{\varepsilon_{p}=-1}\left(1-p^{-s+1 / 2}\right)\right) Z\left(s+\frac{1}{2}\right) Z\left(s-\frac{1}{2}\right) L(s, f) .
$$

We have $\eta_{p} F=F$ for each $p$.

Proof. (i) Let $\pi$ be the cuspidal automorphic representation of $\operatorname{PGL}(2, \mathbb{A})$ corresponding to $f$. In Theorem 1.1 let $S=\{\infty\}$. Since $\varepsilon(1 / 2, \pi)$ is the sign in the functional equation of $L(s, f)$, the sign condition in Theorem 1.1 is fulfilled by our hypothesis. Hence we obtain a cuspidal lifting $\Pi=\Pi\left(\pi \otimes \pi_{S}\right)$. By definition of $S$, the local components $\Pi_{p}$ for $p \mid m$ are either $\Pi(\mathrm{St} \otimes \mathbf{1})$ or $\Pi(\xi \mathrm{St} \otimes \mathbf{1})$, each of which contains an essentially unique paramodular-invariant vector. Together with the lowest weight vector at the archimedean place and the unramified vectors for finite $p \nmid m$, we get a cuspidal Siegel modular form $F \in S_{k}\left(\Gamma^{\text {para }}(m)\right)$. The assertion about the Atkin-Lehner eigenvalues can be read off from table (30), and the $L$-function of $F$ can easily be determined using table (33) (the factor $s-1 / 2$ comes from the archimedean place, see [Sch2], (11)).

As for the uniqueness statement, assume that $F^{\prime}$ is another cusp form with $L$-function as in (35). Let $\Phi^{\prime}$ be the corresponding adelic function, generating (a multiple of) an automorphic representation $\Pi^{\prime}$. From the form of the Euler factors at good primes, we see that the local components of $\Pi$ and of $\Pi^{\prime}$ coincide almost everywhere. Thus $\Pi^{\prime}$ is also 'strongly associated to $P$ " in the terminology of [PS]. By [PS], Theorem 2.2, considering the way the liftings in our Theorem 1.1 are constructed, the representation $\Pi^{\prime}$ is also a lift of the form $\Pi\left(\pi^{\prime} \otimes \pi_{S^{\prime}}\right)$ for some automorphic representation $\pi^{\prime}$ of $\operatorname{GL}(2, \mathbb{A})$ and some set of places $S^{\prime}$ (see the proof of Theorem 1.1).

The local components $\Pi_{p}^{\prime}$ of $\Pi^{\prime}$, being Iwahori-spherical, must therefore be amongst the ones occuring in table (30). A look at the Euler factors (33) of these representations shows that $\Pi_{p}=\Pi_{p}^{\prime}$ for $p \mid m$. Similarly $\Pi_{\infty}=\Pi_{\infty}^{\prime}$. Therefore the global representations $\Pi$ and $\Pi^{\prime}$ are isomorphic. By [PS], Theorem 6.2, the multiplicity one result for lifts from $\widetilde{\mathrm{SL}}(2, \mathbb{A})$, the representations $\Pi$ and $\Pi^{\prime}$ coincide as spaces of automorphic forms. We have shown that $\Phi$ and $\Phi^{\prime}$ are elements of the same irreducible space of automorphic forms. The uniqueness statement now follows from the local uniqueness expressed by the one-dimensionality of the spaces of fixed vectors in (30).

(ii) The proof is similar as in (i). This time we choose $S=\{\infty\} \cup\left\{p \mid m: \varepsilon_{p}=-1\right\}$. Since $\varepsilon(1 / 2, \pi)=(-1)^{k-1} \prod_{p} \varepsilon_{p}=(-1)^{k-1}(-1)^{\# S-1}$, the sign condition in Theorem 1.1 is 
equivalent to our hypothesis that $k$ is even. Hence we get a cuspidal lifting $\Pi=\Pi\left(\pi \otimes \pi_{S}\right)$. By definition of $S$, the local components $\Pi_{p}$ for $p \mid m$ are either $\Pi(\xi \mathrm{St} \otimes \mathbf{1})$ or $\Pi(\mathrm{St} \otimes \mathrm{St})$. In either case, by (30), we have an essentially unique local fixvector for the Siegel congruence subgroup $P_{1}$. We can therefore extract a Siegel cusp form $F \in S_{k}\left(\Gamma_{0}(m)\right)$ from $\Pi$. The Atkin-Lehner eigenvalues and the $L$-function can be seen from the tables (30) and (33). The uniqueness proof is the same as in (i).

Remarks. (a) The factor $s-1 / 2$ in the $L$-functions, which appears because our sets $S$ contain the archimedean place, assures that the $L$-functions are holomorphic at $s=1 / 2$. However, because of the presence of the zeta functions, the $L$-functions of all our lifts have simple poles at $s=3 / 2$ and $s=-1 / 2$.

(b) If $m=1$, then (i) and (ii) are equivalent statements. This is the classical SaitoKurokawa case.

(c) If $m$ is divisible by many primes, a single modular form $f \in S_{2 k-2}\left(\Gamma_{0}(m)\right)$ can have many Saito-Kurokawa lifts, corresponding to various choices of $S$. All that has to be observed is the parity condition in Theorem 1.1. However, most of the liftings thus obtained will be cusp forms with respect to "mixed" congruence subgroups.

(d) As mentioned in the introduction, part (ii) of the theorem holds for arbitrary positive integers $m$, not only square-free ones. This result can be reproved using the above methods, which requires a more delicate analysis of the local representations. The necessary invariance properties were obtained in collaboration with Brooks Roberts and will appear elsewhere.

(e) If we choose $S$ not to contain the archimedean place, we obtain certain nonholomorphic Siegel modular forms. See $[\mathrm{Mz}]$ for a construction using classical notation.

Examples. (a) There exists an elliptic eigenform $f \in S_{2}^{\text {new }}\left(\Gamma_{0}(37)\right)$ with AtkinLehner eigenvalue $\varepsilon_{37}=1$. Since $2=2 \cdot 2-2$, both sign conditions in (i) and (ii) of Theorem 5.2 are fulfilled, and we get Siegel modular forms $F_{1} \in S_{2}\left(\Gamma^{\text {para }}(37)\right)$ and $F_{2} \in S_{2}\left(\Gamma_{0}(37)\right)$. In this case $F_{1}$ and $F_{2}$ correspond to different vectors in the same automorphic representation of $\operatorname{PGSp}(4, \mathbb{A})$; the local representation at the place 37 is $\Pi(\xi \mathrm{St} \otimes \mathbf{1})$.

(b) There exists an elliptic eigenform $f \in S_{2}^{\text {new }}\left(\Gamma_{0}(91)\right)$ with Atkin-Lehner eigenvalues $\varepsilon_{7}=\varepsilon_{13}=-1$. Again both sign conditions are fulfilled, so that we get two Siegel modular forms $F_{1} \in S_{2}\left(\Gamma^{\text {para }}(91)\right)$ and $F_{2} \in S_{2}\left(\Gamma_{0}(91)\right)$. In this case the two modular forms are vectors in different (but near equivalent) automorphic representations of $\operatorname{PGSp}(4, \mathbb{A})$.

Finally, let us compare the construction in part (i) of Theorem 5.2 with the Yoshida liftings of [Yo1], [Yo2] and [BSP1], [BSP2]. The starting point for the latter is also an eigenform $f \in S_{2 k-2}\left(\Gamma_{0}(m)\right)$ of some square-free level $m$. The first step is to move $f$ to an automorphic form $\varphi$ on the unit group $D^{*}(\mathbb{A})$ of a global quaternion algebra $D$ via the Jacquet-Langlands correspondence. Next, the close relationship between $D^{*}(\mathbb{A})$ and global orthogonal groups is exploited to produce an automorphic form $\phi$ on some $\operatorname{GO}(V, \mathbb{A})$, where $V$ is a four-dimensional quadratic space (this corresponds to the left vertical arrows in the diagram (12) in [Sch2]). This function is then moved to a Siegel modular form (an 
automorphic form on $\operatorname{GSp}(4, \mathbb{A}))$ via the theta correspondence. The result is a cusp form of degree 2 of the same level $m$ (meaning for $\Gamma_{0}(m)$ ).

To make this procedure work, one has to choose a suitable quaternion algebra $D$. Apparently $D$ has to be ramified at $\infty$, and then necessarily also at an odd number of finite places $p$. But $D$ must be unramified at finite places outside $m$, because otherwise $f$ has no Jacquet-Langlands lift. It follows that necessarily $m \neq 1$, i.e., classical Saito-Kurokawa lifts are not Yoshida lifts.

Let us assume that $m>1$ and that $D$ is chosen such that the Jacquet-Langlands lift $\varphi$ of $f$ exists. Then another assumption had to be made in [BSP1] and [BSP2] to obtain a non-vanishing function $\phi$ on the orthogonal group, namely, for all $p \mid m$ we must have

$$
D \text { unramified at } p \quad \Leftrightarrow \quad \varepsilon_{p}=1
$$

Thus $f$ completely determines $D$. For fixed $f$, there is at most one Yoshida lift. Note that the condition (36) amounts precisely to the choice of $S$ made in part (i) of the theorem above. The unique Saito-Kurokawa lift $F$ for $\Gamma_{0}(m)$ constructed in this theorem coincides with the Yoshida lift of $f$. The weight condition that $k$ be even is also necessary in [BSP1] and [BSP2], namely for the final theta lifting to be non-zero.

Thus the point is that our Saito-Kurokawa lifts are locally lifted from orthogonal groups (see [Sch2]), while this is true globally for Yoshida lifts.

\section{References}

[An] Andrianov, A., Modular descent and the Saito-Kurokawa conjecture, Invent. Math. 53 (1979), $267-280$.

[AS] Asgari, M., Schmidt, R., Siegel modular forms and representations, Manuscr. Math. 104 (2001), 173200.

[BSP1] Böcherer, S., Schulze-Pillot, R., Siegel modular forms and theta series attached to quaternion algebras, Nagoya Math. J. 121 (1991), 35-96.

[BSP2] Böcherer, S., Schulze-Pillot, R., Siegel modular forms and theta series attached to quaternion algebras, II, Nagoya Math. J. 147 (1997), 71-106.

[Bu] Bump, D., Automorphic Forms and Representations, Cambridge University Press, 1996.

[EZ] Eichler, M., Zagier, D., The Theory of Jacobi Forms, Birkhäuser, Progr. Math. 55, Basel 1985.

[Fr] Freitag, E., Siegelsche Modulfunktionen, Springer-Verlag, 1983.

[Ge] Gelbart, S., Automorphic Forms on Adele Groups, Ann. Math. Stud. 83, Princeton 1975.

[Gr] Gritsenko, V., Arithmetical lifting and its applications, in: Number theory (Paris 1992-1993), London Math. Soc. Lecture Note Ser. 215, Cambridge Univ. Press, Cambridge (1995), 103-126.

[KL] Kazhdan, D., Lusztig, G., Proof of the Deligne-Langlands conjecture for Hecke algebras, Invent. Math. 87 (1987), 153-215.

[KPS] Kim, J.-L., Piatetski-Shapiro, I., Quadratic base change of $\theta_{10}$, Israel J. Math. 123 (2001), 317-340.

$[\mathrm{Ku}]$ Kurokawa, N., Examples of eigenvalues of Hecke operators on Siegel cusp forms of degree two, Invent. Math. 49 (1978), 149-165.

[La] Langlands, R., Automorphic representations, Shimura varieties, and motives. Ein Märchen, Proc. Sympos. Pure Math. 33 (1979), part 2, 205-246.

[Ma] Maass, H., Über eine Spezialschar von Modulformen zweiten Grades I, II, III, Invent. Math. 52 (1979), 95-104; Invent. Math. 53 (1979), 249-253; Invent. Math. 53 (1979), 255-265.

[MR1] Manickam, M., Ramakrishnan, B., On Shimura, Shintani and Eichler-Zagier correspondences, Trans. Amer. Math. Soc. 352 (2000), 2601-2617.

[MR2] Manickam, M., Ramakrishnan, B., On Saito-Kurokawa correspondence of degree two for arbitrary level, J. Ramanujan Math. Soc. 17 (2002), 149-160. 
[MRV] Manickam, M., Ramakrishnan, B., Vasudevan, T., On Saito-Kurokawa descent for congruence subgroups, Manuscr. Math. 81 (1993), 161-182.

[Mi] Miyake, T., Modular Forms, Springer-Verlag, 1989.

[Mz] Miyazaki, T., On Saito-Kurokawa lifting to cohomological Siegel modular forms, Manuscr. Math. 114 (2004), 139-163.

[PS] Piatetski-Shapiro, I., On the Saito-Kurokawa lifting, Invent. Math. 71 (1983), 309-338.

[ST] Sally, P., Tadić, M., Induced representations and classifications for $\operatorname{GSp}(2, F)$ and $\operatorname{Sp}(2, F)$, Bull. Soc. Math. France 121, Mem. 52 (1993), 75-133.

[Sch1] Schmidt, R., Some remarks on local newforms for GL(2), J. Ramanujan Math. Soc. 17 (2002), $115-147$.

[Sch2] Schmidt, R., The Saito-Kurokawa lifting and functoriality, Amer. J. Math. 127 (2005), 209-240.

[Sch3] Schmidt, R., Iwahori-spherical representations of GSp(4) and Siegel modular forms of degree 2 with square-free level, J. Math. Soc. Japan 57 (2005), 259-293.

[SZ] Skoruppa, N.-P., Zagier, D., Jacobi forms and a certain space of modular forms, Invent. Math. 94 (1988), 113-146.

[Wa1] Waldspurger, J.-L., Correspondance de Shimura, J. Math Pures Appl. 59 (1980), 1-133.

[Wa2] Waldspurger, J.-L., Correspondance de Shimura et quaternions, Forum Math. 3 (1991), 219-307.

[Yo1] Yoshida, H., Siegel's modular forms and the arithmetic of quadratic forms, Invent. Math. 60 (1980), 193-248.

[Yo2] Yoshida, H., On Siegel modular forms obtained from theta series, J. reine angew. Math. 352 (1984), 184-219.

[Yo3] Yoshida, H., The action of Hecke operators on theta series, in: Algebraic and topological theories-to the memory of Dr. T. Miyata (1985), 197-238.

[Za] Zagier, D., Sur la conjecture de Saito-Kurokawa (d'après H. Maass), in: Seminar on Number Theory, Paris 1979-80, Birkhäuser, Progr. Math. 12, Boston, Mass. (1981), 371-394.

Department of Mathematics, University of Oklahoma, Norman, OK 73019-0315 e-mail: rschmidt@math.ou.edu

Eingegangen 17. Dezember 2003, in revidierter Fassung 12. Februar 2006 